\title{
The evolution of a front in turbulent thermal wind balance, Part I: Theory
}

\author{
Matthew N. Crowe and John R. Taylor \\ Department of Applied Mathematics and Theoretical Physics, University of Cambridge, \\ Centre for Mathematical Sciences, Wilberforce Road, Cambridge, CB3 0WA, UK
}

(Received xx; revised xx; accepted xx)

Here, we examine the influence of small-scale turbulence on the evolution of fronts in the ocean and atmosphere. Specifically, we consider the evolution of an initially balanced density front, subject to an imposed viscosity and diffusivity as a simple analogue for small-scale turbulence. At late times, the dominant balance is found to be the quasi-steady Turbulent Thermal Wind (TTW) balance with time-evolution due to an advection-diffusion balance in the buoyancy equation. We use the leading order balance to determine analytical similarity solutions for the spreading of a front and find that the spreading rate is maximum for an intermediate value of the Ekman number with the spreading resulting from shear dispersion associated with the cross-front flow and vertical diffusion of density. In response to shear dispersion, the front evolves towards a density profile that is nearly linear in the cross-front coordinate. At the edges of the frontal zone, the density field develops large curvature and these regions are associated with narrow bands of intense vertical velocity.

\section{Introduction}

Fronts, or regions with large horizontal density gradients, are common and important features in the atmosphere and ocean. Atmospheric fronts are associated with weather patterns on both the local (Ostdiek \& Blumen 1997) and the continental scale (Hoskins 1982) and coincide with strong winds and updrafts. Similarly, fronts in the upper ocean can enhance the vertical transport of tracers such as heat and nutrients (Garrett \& Loder 1981; Ferrari 2011).

Fronts develop and intensify through a process known as frontogenesis (Hoskins 1982). This phenomenon occurs in both the atmosphere and oceans and can be driven by a variety of processes including externally imposed strain, surface heat flux, wind stress and adjustment from an unbalanced initial state (Hoskins \& Bretherton 1972; Blumen 2000). Large scale fronts in the ocean and atmosphere are often close to a state of thermal wind balance; the balance between a pressure gradient in hydrostatic balance with changes in density and the Coriolis force associated with an along-front jet (Holton \& Hakim 2012; Rudnick \& Luyten 1996). When a balanced front is disturbed (for example by turbulent mixing, large scale flow, or surface stress), the dynamic response results in a secondary circulation with flow in the cross-front and vertical directions (Eliassen 1962; Hoskins \& Bretherton 1972; Orlanski \& Ross 1977). The horizontal convergence associated with the secondary circulation further intensifies frontogenesis (Hoskins \& Bretherton 1972).

Inviscid analytic solutions for fronts subject to an imposed convergent strain flow can develop singularities in finite time (Hoskins \& Bretherton 1972). These infinitely sharp fronts are not observed physically, suggesting the existence of a process controlling frontal width. One candidate for arresting frontogenesis is mixing of density and momentum by small scale turbulence (McWilliams 2016; Sullivan \& McWilliams 2018). 
The evolution of a front in response to frictional forcing was considered by Thompson (2000) who used a two-dimensonal semi-geostrophic model modified to include vertical mixing. A constant diffusivity and viscosity was used to represent turbulent effects in the ocean surface mixed layer. A cross-front ageostrophic flow proportional to the horizontal buoyancy gradient led to a slumping of the frontal region and the formation of a sharp surface buoyancy gradient. In the case where vertical diffusion was small a buoyancy balance between time evolution and cross-front advection was considered giving an equation for the non-linear evolution of the surface buoyancy. The solution evolved towards a finite-time discontinuity as with the inviscid case and gave a good approximation to the frontal sharpening before the singularity was reached.

Early theoretical work by Garrett \& Loder (1981) considered an idealised model of a front for two-layer and continuous stratification. For a continuously stratified front with small Ekman number it was assumed that the along-front velocity satisfies thermal wind balance allowing the cross-front velocity to be approximated using an eddy viscosity acting on the thermal wind flow. Using the equation for density advection and neglecting eddy diffusivity, a diffusion equation with variable horizontal diffusivity was found for the depth of isopycnals outside of the surface Ekman layer.

Later Young (1994) derived the subinertial mixed layer (SML) approximation using a simple vertical mixing parametrisation which assumed that quantities evolve towards their depth averaged value at a rate linearly proportional to their departure from this depth average. Separate timescales for vertical mixing and inertia were considered and analytic solutions were given in terms of their ratio under the assumption of small Rossby number. In the case of fast momentum mixing, temperature and salinity were found to satisfy nonlinear advection-diffusion equations with an effective diffusivity proportional to the square of the buoyancy gradient. In this case, the effective diffusivity was due to shear dispersion associated with a vertically-sheared cross-front flow and vertical diffusion.

In the limit of rapid mixing, the dominant balance in the SML approximation is between the hydrostatic pressure gradient, the Coriolis force and the vertical mixing of momentum. This quasi-steady balance, recently termed the 'Turbulent thermal wind' (TTW) or 'generalised Ekman' has been seen in models and observations of ocean fronts (Cronin \& Kessler 2009; Taylor \& Ferrari 2010; Gula et al. 2014; McWilliams et al. 2015; Wenegrat \& McPhaden 2016) where the vertical momentum fluxes are associated with small-scale turbulence. As we will show, TTW balance arises as the small Ro and small aspect ratio limit, corresponding to a shallow, strongly rotating flow, as described earlier by Charney (1973).

McWilliams (2017) extended the previous analysis by combining the generalised nonlinear TTW equations with an Omega equation $(\Omega \mathrm{E})$ to construct a diagnostic framework to analyze the frontogenetic tendency and secondary circulation for fronts and filaments. For a given buoyancy field, the velocity fields were decomposed into TTW and $\Omega \mathrm{E}$ components and an iterative procedure was used to numerically converge to the diagnostic secondary circulation. This procedure allowed the velocity field to be diagnosed for an imposed buoyancy field, boundary conditions, and an externally imposed strain flow. By diagnosing the time-tendency, McWilliams (2017) showed that the ageostrophic secondary circulation associated with TTW balance could drive frontogenesis. While this analysis allowed the diagnosis of the instantaneous time tendency, it did not examine the temporal evolution of the front.

Other recent studies have examined the evolution of submesoscale filaments in TTW balance using numerical simulations with parameterized (McWilliams et al. 2015) and partially-resolved (Sullivan \& McWilliams 2018) turbulence. These studies found that the TTW circulation is associated with convergent surface flow and downwelling along 
the centre of the filament. This induces frontogenesis which is eventually arrested by small-scale turbulence (Sullivan \& McWilliams 2018).

Our objective is to develop a theoretical description of the evolution of a front in TTW balance. This can be viewed as an extension to the diagnostic analysis by McWilliams (2017), although we restrict our analysis to small Rossby numbers where TTW balance holds. However, our analysis does extend to large Ekman number and in this way our study can be viewed as a generalisation of the Garrett \& Loder (1981) model. For simplicity, we represent small-scale turbulence through a prescribed viscosity and diffusivity which are allowed to vary in depth but are assumed to be independent of time and both horizontal directions.

We find that frontogenesis, or intensification of the surface density gradient, occurs during a brief transient 'adjustment' period. After this transient period, we find that the front spreads through shear dispersion associated with the vertical diffusivity and the vertically-sheared cross-front velocity, as described in Young (1994). Here, our focus will be on the spreading regime, rather than the frontogenetic regime, conditions for which were examined in detail in McWilliams (2017). In a region of frontogenesis the cross front length scales can become small corresponding to an order one Rossby number and advection by the secondary circulation at leading order, we instead consider an unforced front with no sharp surface gradients in which the horizontal length scale can be taken as the frontal width and the resulting Rossby number is small.

Further, we find that when the viscosity and diffusivity are sufficiently large, the front spreads through shear dispersion associated with the vertical diffusivity and the vertical shear of the cross-front velocity, a process also described by Young (1994) in the context of the SML equations. Further, we find that the density evolves towards a self-similar profile with density being an approximately piecewise linear function of cross-front distance with a relatively constant density gradient inside the front and large curvature at the edges of the front. The vertical velocity is intensified in the regions of large curvature at the edges of the front, consistent with the instantaneous TTW balance associated with the self-similar density profile.

In $\S 2$ we describe the problem setup and our approach to finding a solution using an expansion in Rossby number and a multiple time scale analysis. In $\S 3.1-\S 3.3$ we consider the balances at $O(1), O(R o)$ and $O\left(R o^{2}\right)$ which we find are sufficient to determine a solution to leading order in velocity and first order in buoyancy. We find that the governing equation for the depth-averaged buoyancy can be solved using a similarity solution which we calculate in $\S 3.5$. Analytic solutions for velocity and buoyancy are found in terms of vertical structure functions. These can be solved for analytically for simple turbulence parametrisations while numerical solutions are required in general. Finally in $\S 4$ we discuss our results and future work.

\section{Problem Setup}

We will consider an idealised frontal geometry consisting of an incompressible fluid bounded from above and below by rigid, horizontal surfaces in a reference frame rotating about the vertical $(z)$ axis. The fluid density varies continuously across a single isolated front. This geometry is a canonical configuration for studies of frontal dynamics (e.g. Hoskins \& Bretherton (1972); Blumen (2000)). For simplicity, we neglect the horizontal component of the rotation vector, the so-called 'traditional approximation' (Salmon 1998).

We will assume that density changes can be represented by a single scalar equation, invoking a linear equation of state, and that variations in density are small compared 
to a reference value, invoking the Boussinesq approximation. We will let $b \equiv-g \rho^{\prime} / \rho_{0}$ denote the fluid buoyancy, where $\rho_{0}$ is a constant reference density, $\rho^{\prime}$ denotes departures from $\rho_{0}$, and $\rho^{\prime}<<\rho_{0}$.

We will consider the response of an initially balanced front to an imposed viscosity $\nu$ and diffusivity $\kappa$ which we assume depend only on depth, $z$. Note that, while $\nu$ and $\kappa$ will be assumed to be constant in time, our primary motivation is to study the influence of small-scale turbulence on the evolution of the front. By using a time-independent $\nu$ and $\kappa$, we are able to isolate the influence of a turbulent viscosity and diffusivity on the evolution of the front, without allowing the feedback associated with the front altering the properties of small-scale turbulence. This assumption is artificial but greatly simplifies the analysis. When considering constant $\nu$ and $\kappa$ (as in Thompson (2000)), the setup can also be viewed as a laboratory scale analogue with molecular $\nu$ and $\kappa$.

We can non-dimensionalise the governing equations using a horizontal length scale $L$, vertical length scale $H$, and buoyancy scale $\Delta b$, with the horizontal velocity scale $U=\Delta b H /(f L)$, vertical velocity scale $W=U H / L=\Delta b H^{2} /\left(f L^{2}\right)$, pressure scale $P=f U L=\Delta b H$, and timescale $T=L / U=f L^{2} /(H \Delta b)$ for Coriolis parameter $f$. We define the Rossby number, $R o=U /(f L)$, using the geostrophic shear, $U / H=\partial b / \partial x / f=$ $\Delta b /(f L)$ and write the aspect ratio, $H / L$, as $\epsilon$. The Ekman number and Prantl number are defined in terms of the depth averaged dimensional viscosity and diffusivity, $\bar{\nu}$ and $\bar{\kappa}$, as $E=\bar{\nu} /\left(f H^{2}\right)$ and $\operatorname{Pr}=\bar{\nu} / \bar{\kappa}$. This gives the following non-dimensional equations (Charney 1973):

$$
\begin{aligned}
R o \frac{D u}{D t}-v & =-\frac{\partial p}{\partial x}+E \nabla_{\epsilon} \cdot\left(\nu \nabla_{\epsilon} u\right) \\
R o \frac{D v}{D t}+u & =-\frac{\partial p}{\partial y}+E \nabla_{\epsilon} \cdot\left(\nu \nabla_{\epsilon} v\right), \\
R o \epsilon^{2} \frac{D w}{D t} & =-\frac{\partial p}{\partial z}+b+\epsilon^{2} E \nabla_{\epsilon} \cdot\left(\nu \nabla_{\epsilon} w\right), \\
R o \frac{D b}{D t} & =\frac{E}{P r} \nabla_{\epsilon} \cdot\left(\kappa \nabla_{\epsilon} b\right), \\
\frac{\partial u}{\partial x}+\frac{\partial v}{\partial y}+\frac{\partial w}{\partial z} & =0
\end{aligned}
$$

for parameters defined in Table 1 . The viscosity and diffusivity functions, $\nu(z)$ and $\kappa(z)$, depend only on depth, $z$, and are normalised by their depth-averaged values, $\bar{\nu}$ and $\bar{\kappa}$, respectively. The operators $D / D t$ and $\nabla_{\epsilon}$ are given by

$$
\begin{aligned}
\frac{D}{D t} & =\frac{\partial}{\partial t}+u \frac{\partial}{\partial x}+v \frac{\partial}{\partial y}+w \frac{\partial}{\partial z} \\
\nabla_{\epsilon} & =\left(\epsilon \frac{\partial}{\partial x}, \epsilon \frac{\partial}{\partial y}, \frac{\partial}{\partial z}\right)
\end{aligned}
$$

In the following analysis, we will allow arbitrary values of $\mathrm{Pr}$, but will highlight the case where $\operatorname{Pr}=1$. We use boundary conditions with vanishing vertical shear, vertical velocity, and buoyancy flux on the top and bottom boundaries and consider a fluid layer between $z= \pm 0.5$ for unbounded $x$ and $y$.

We will later consider the two dimensional case where the $x$-axis is aligned with the local density (or buoyancy) gradient. Motivated by the observation that variations aligned with the frontal axis are much smaller than cross-front variations, we will neglect alongfront derivatives, although we will retain all three components of the velocity vector. We 
Parameter Rossby No. Ekman No. Prandtl No. Aspect Ratio

$\begin{array}{ccccc}\text { Symbol } & R o & E & P r & \epsilon \\ \text { Definition } & \epsilon \Delta b / f^{2} L & \bar{\nu} / f H^{2} & \bar{\nu} / \bar{\kappa} & H / L\end{array}$

Table 1: Definitions of the dimensionless parameters and their values for buoyancy difference, $\Delta b$, Coriolis parameter, $f$, horizontal lengthscale, $L$, vertical lengthscale, $H$ and depth-averaged dimensional viscosity and diffusivity, $\bar{\nu}$ and $\bar{\kappa}$.

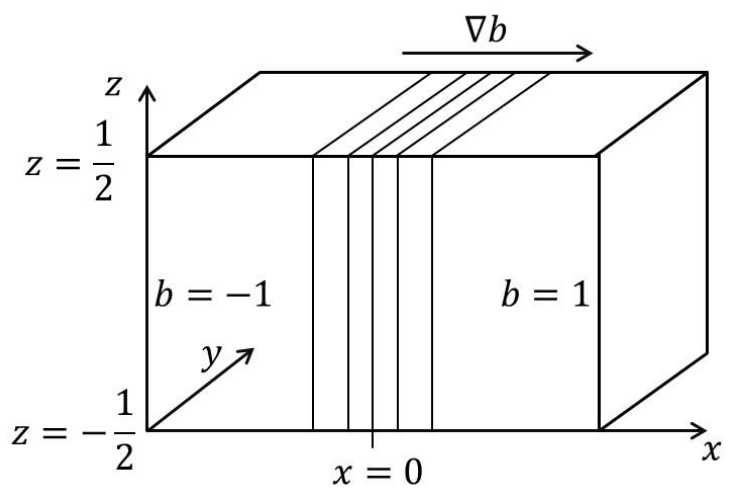

Figure 1: Non-dimensional frontal geometry.

then assume without loss of generality that the low buoyancy region is on the left of the front while the high buoyancy region is on the right (see Figure 1).

\section{Analytic solution}

Here, we analyze Eqns. 2.1 using a multiple timescale asymptotic analysis with $R o$ as our small parameter and $\epsilon=O(R o)$. Unlike the Rossby number, we do not assume that the Ekman number is small, and specifically consider $E=O(1)$. Later, in Section 3.4 we will discuss the limit of $E=O(R o)$ and the connection with previous work by Garrett \& Loder (1981).

After expanding $\mathbf{u}$ and $b$ in powers of $R o$, we find that there are two relevant timescales: a fast timescale, $\tau=t / R o$, representing vertical diffusion and initial adjustment, and a slow timescale, $T=R o t$, representing horizontal shear dispersion and spreading. The intermediate timescale, $t$, does not appear in the analysis.

We can use these new timescales to expand the time derivative as

$$
\frac{\partial}{\partial t}=\frac{1}{R o} \frac{\partial}{\partial \tau}+R o \frac{\partial}{\partial T}
$$

The velocity and buoyancy are also expanded in powers of $R o$,

$$
\mathbf{u}=\mathbf{u}_{0}+R o \mathbf{u}_{1}+\ldots, \quad \text { and } b=b_{0}+R o b_{1}+\ldots
$$

For simplicity, we restrict our attention to initial conditions where the buoyancy is independent of depth, i.e. $\left.b\right|_{t=0}=f(x, y)$, although the initial velocity can have arbitrary depth dependence. After using the expansions introduced above, we collect terms in the governing equations (2.1) of like order in Ro. The sections below describe solutions of the resulting equations for the leading order contributions to velocity and buoyancy. 


\section{1. $\mathbf{O}(\mathbf{1})$ Balance}

We now consider the leading order buoyancy and momentum balances with $O(1)$ terms. The steady version of this balance is the turbulent thermal wind (TTW) balanced described by Gula et al. (2014); McWilliams (2017). The analysis in this section extends their work by including time dependence and unbalanced initial conditions. We find that the time dependence occurs on the fast timescale, $\tau$, and that as $\tau \rightarrow \infty$, the steady TTW balance is recovered.

\subsubsection{Buoyancy}

The $O(1)$ terms in the buoyancy conservation equation $(2.1 d)$ are

$$
\frac{\partial b_{0}}{\partial \tau}=\frac{E}{\operatorname{Pr}} \frac{\partial}{\partial z}\left(\kappa \frac{\partial b_{0}}{\partial z}\right) .
$$

This is a diffusion equation and since the initial state is depth independent the solution is of the form

$$
b_{0}=b_{0}(x, y, T) \quad \text { where } \quad b_{0}(x, y, 0)=f(x, y),
$$

which is vertically homogeneous and hence not affected by vertical diffusion. We note that a $z$ dependent initial buoyancy field will result in diffusion towards a vertically homogeneous state on the fast timescale $\tau$, hence the leading order buoyancy will be depth independent after some fast initial adjustment. Taking a depth independent initial $b_{0}$ will not change the long term behaviour of the system and will just add additional transients to the velocity fields and higher order buoyancy terms.

\subsubsection{Velocity}

The $O(1)$ terms in the momentum and continuity equations $(2.1 a-2.1 c$ and $2.1 e)$ are

$$
\begin{aligned}
\frac{\partial u_{0}}{\partial \tau}-v_{0} & =-\frac{\partial p_{0}}{\partial x}+E \frac{\partial}{\partial z}\left(\nu \frac{\partial u_{0}}{\partial z}\right), \\
\frac{\partial v_{0}}{\partial \tau}+u_{0} & =-\frac{\partial p_{0}}{\partial y}+E \frac{\partial}{\partial z}\left(\nu \frac{\partial v_{0}}{\partial z}\right), \\
-\frac{\partial p_{0}}{\partial z}+b_{0} & =0 \\
\frac{\partial u_{0}}{\partial x}+\frac{\partial v_{0}}{\partial y}+\frac{\partial w_{0}}{\partial z} & =0 .
\end{aligned}
$$

Equation $3.5 c$ is hydrostatic balance, and since $b_{0}$ is independent of $z$, this gives

$$
p_{0}=b_{0} z
$$

using $z=0$ as the reference level. Note that the $O(1)$ momentum equations are linear in the horizontal velocity, $\mathbf{u}_{H 0}=\left(u_{0}, v_{0}, 0\right)$. Through the hydrostatic relation, the horizontal pressure gradient is a linear function of the horizontal buoyancy gradient. Therefore, the horizontal velocity is a linear function of the horizontal buoyancy gradient, i.e.

$$
\left(\begin{array}{l}
u_{0} \\
v_{0}
\end{array}\right)=-\sqrt{E}\left(\begin{array}{ll}
A_{1}(\zeta, \tau) & A_{2}(\zeta, \tau) \\
B_{1}(\zeta, \tau) & B_{2}(\zeta, \tau)
\end{array}\right)\left(\begin{array}{l}
\frac{\partial b_{0}}{\partial x} \\
\frac{\partial b_{0}}{\partial y}
\end{array}\right)
$$


where $\zeta=z / \sqrt{E}$. Splitting equations $3.5 a$ and $3.5 b$ into parts linear in $\partial b_{0} / \partial x$ and $\partial b_{0} / \partial y$ gives the system of equations

$$
\begin{aligned}
& L_{\nu} A_{1}=B_{1}+\zeta, \\
& L_{\nu} A_{2}=B_{2}, \\
& L_{\nu} B_{1}=-A_{1}, \\
& L_{\nu} B_{2}=-A_{2}+\zeta,
\end{aligned}
$$

where $L_{\nu}=\frac{\partial}{\partial \tau}-\frac{\partial}{\partial \zeta}\left(\nu \frac{\partial}{\partial \zeta}\right)$ is a diffusion operator. These equations can be combined to give

$$
-L_{\nu}^{2} K(\zeta, \tau)=K(\zeta, \tau)+\zeta
$$

and $K(\zeta, \tau)$ is a function to be determined. Applying stress free boundary conditions at $z= \pm 1 / 2$ gives $B_{1}=-A_{2}=K$ and $A_{1}=B_{2}=-L_{\nu} K$. The $O(1)$ horizontal velocity can then be written

$$
\mathbf{u}_{H 0}=-\sqrt{E} \mathbf{K}(\zeta, \tau) \cdot \nabla_{H} b_{0},
$$

where

$$
\mathbf{K}=\left(\begin{array}{cc}
-L_{\nu} K & -K \\
K & -L_{\nu} K
\end{array}\right)
$$

and $\nabla_{H}$ is the horizontal gradient operator. It is instructive to re-write Eq. (3.10) as

$$
\mathbf{u}_{H 0}=-\sqrt{E}\left[-L_{\nu} K \nabla_{H} b_{0}+K \hat{\mathbf{z}} \times \nabla_{H} b_{0}\right],
$$

hence $-L_{\nu} K$ describes the velocity component that is locally perpendicular to the front while $K$ describes the velocity that is locally orientated along the front.

From mass conservation we can solve for $w_{0}$ in terms of $K(\zeta, \tau)$ :

$$
\frac{\partial w_{0}}{\partial z}=-\nabla_{H} \cdot \mathbf{u}_{H 0}=-\sqrt{E} L_{\nu} K\left(z^{\prime}, \tau\right) \nabla_{H}^{2} b_{0},
$$

which can be integrated to obtain

$$
w_{0}=-\sqrt{E}\left(\int_{-1 / 2}^{z} L_{\nu} K\left(z^{\prime}, \tau\right) d z^{\prime}\right) \nabla_{H}^{2} b_{0} .
$$

To complete the solution for the $O(1)$ velocity, we need to solve for $K(\zeta, \tau)$. We begin by considering the steady state solution obtained by neglecting the time derivatives in equations $3.5 a$ and $3.5 b$ and denoting the steady state solution $K_{0}(\zeta)$. Equation 3.9 becomes

$$
-\frac{\partial}{\partial \zeta}\left(\nu \frac{\partial^{2}}{\partial \zeta^{2}}\left(\nu \frac{\partial}{\partial \zeta} K_{0}\right)\right)=K_{0}+\zeta .
$$

For a general $\nu(\zeta)$ we can solve this equation numerically, subject to boundary conditions $K_{0}^{\prime}=0$ and $\left(\nu K_{0}^{\prime}\right)^{\prime \prime}=0$ at $z= \pm 0.5$. The steady state vertical velocity is given by

$$
w_{0}=E \nu(z) K_{0}^{\prime}(z / \sqrt{E}) \nabla_{H}^{2} b_{0} .
$$

We now return to the general time dependent case described by equation 3.9 and let $K(\zeta, t)=K_{1}(\zeta, t)+K_{0}(\zeta)$ for steady state solution $K_{0}$. Equation 3.9 becomes

$$
-\frac{\partial}{\partial \zeta}\left(\nu \frac{\partial^{2}}{\partial \zeta^{2}}\left(\nu \frac{\partial}{\partial \zeta} K_{0}\right)\right)-L_{\nu}^{2} K_{1}=K_{0}+K_{1}+\zeta
$$


hence

$$
-L_{\nu}^{2} K_{1}=K_{1}
$$

with boundary conditions $K_{1}^{\prime}=\left(L_{\nu} K_{1}\right)^{\prime}=0$ at $z= \pm 0.5$. Substituting for $L_{\nu}$ gives

$$
\left(\frac{\partial}{\partial \tau}-E \frac{\partial}{\partial z} \nu \frac{\partial}{\partial z}\right)^{2} K_{1}=-K_{1}
$$

which we solve using separation of variables. Seeking solutions of the form $K_{1}=$ $\mathcal{T}(\tau) \mathcal{Z}(z)$, we find

$$
K_{1}=\sum_{n=0}^{\infty} B_{n} e^{-E \lambda_{n} \tau}\left[\cos \tau+E \lambda_{n} \sin \tau\right] \mathcal{Z}_{n}(z),
$$

where $\mathcal{Z}_{n}$ is an eigenfunction of the linear system

$$
\frac{\partial}{\partial z}\left(\nu \frac{\partial \mathcal{Z}_{n}}{\partial z}\right)=-\lambda_{n} \mathcal{Z}_{n}
$$

with eigenvalue $\lambda_{n}$. The boundary conditions discretise the modes and lead to an orthogonality relation

$$
\int_{-1 / 2}^{1 / 2} \mathcal{Z}_{n} \mathcal{Z}_{m} d z=z_{n}^{2} \delta_{n m}
$$

We now consider initial conditions for $K=K_{0}+K_{1}$ and use Eq. (3.22) to determine the coefficients, $B_{n}$, for each case. Denote the initial conditions, $K(z, 0)=\mathcal{K}(z)$. Using equation 3.20 we have

$$
K_{1}(z, 0)=\sum_{n=0}^{\infty} B_{n} \mathcal{Z}_{n}(z)
$$

and using Eq. (3.22), we have

$$
B_{n}=\frac{1}{z_{n}^{2}} \int_{-1 / 2}^{1 / 2} K_{1}(z, 0) \mathcal{Z}(z) d z=\frac{1}{z_{n}^{2}} \int_{-1 / 2}^{1 / 2}\left[\mathcal{K}(z)-K_{0}(z / \sqrt{E})\right] \mathcal{Z}(z) d z .
$$

Combining the results given above, we can now write out the solution for $K(\zeta, \tau)$,

$$
K(z, \tau)=K_{0}(z / \sqrt{E})+\sum_{n=0}^{\infty} B_{n} e^{-E \lambda_{n} \tau}\left[\cos \tau+E \lambda_{n} \sin \tau\right] \mathcal{Z}_{n}(z),
$$

and solution for $-L_{\nu} K$,

$$
-L_{\nu} K(z, \tau)=E \frac{\partial}{\partial z}\left(\nu \frac{\partial K_{0}}{\partial z}\right)+\sum_{n=0}^{\infty} B_{n} e^{-E \lambda_{n} \tau}\left[\sin \tau-E \lambda_{n} \cos \tau\right] \mathcal{Z}_{n}(z) .
$$

Some important initial conditions include stationary flow $(\mathbf{u}=0)$ which corresponds to $\mathcal{K}=0$, thermal wind balance which corresponds to $\mathcal{K}=-z / \sqrt{E}$, and steady TTW balance which corresponds to $\mathcal{K}=K_{0}$ and hence $B_{n}=0$. In all cases, after an initial adjustment period, the solution for $\mathbf{u}_{0}$ approaches steady TTW balance. The transients decay exponentially on a timescale of $\tau=O(1 / E)$ corresponding to $t=O\left(R_{o} / E\right)$.

\subsubsection{Summary of $O(1)$ solution}

When expanded in the limit of small Rossby number, the buoyancy conservation equation at leading order $(O(1))$ reduces to a diffusion equation on the fast timescale, $\tau$. 
In summary, the $O(1)$ velocity is

$$
\begin{aligned}
& u_{0}=-\sqrt{E}\left[-L_{\nu} K(z, \tau) \frac{\partial b_{0}}{\partial x}-K(z, \tau) \frac{\partial b_{0}}{\partial y}\right], \\
& v_{0}=-\sqrt{E}\left[K(z, \tau) \frac{\partial b_{0}}{\partial x}-L_{\nu} K(z, \tau) \frac{\partial b_{0}}{\partial y}\right],
\end{aligned}
$$

where $K$ and $-L_{\nu} K$ are given by equations 3.25 and 3.26 and

$$
w_{0}=E\left[\nu K_{0}^{\prime}(z / \sqrt{E})-\sum_{n=0}^{\infty} \frac{B_{n}}{\sqrt{E}} e^{-E \lambda_{n} \tau}\left[-\sin \tau+E \lambda_{n} \cos \tau\right] \int_{-1 / 2}^{z} \mathcal{Z}_{n}\left(z^{\prime}\right) d z^{\prime}\right] \nabla_{H}^{2} b_{0},
$$

which reduces to steady TTW balance (Eq. 3.16), for large $\tau$. The $\cos \tau$ and $\sin \tau$ terms correspond to inertial waves generated by departures from steady TTW balance in the initial conditions. Analytic solutions can be obtained for constant $\nu$ (see Appendix A).

It is useful to consider two limiting cases of the $O(1)$ velocity in terms of Ekman number, $E$. For small $E$ the along front velocity approaches thermal wind balance and all other velocity components become small, while for large $E$ all velocity components become small due to the damping of motion by the high viscosity. The dependence of $K_{0}$, and hence $\mathbf{u}_{0}$ and $\psi_{0}$, on Ekman number for a constant $\nu$ is given in Appendix A.

Figure 2 shows the steady state $O(1)$ velocity field (i.e. TTW balance) expressed in terms of an $x-z$ streamfunction, $\psi_{0}$, for a $y$-independent buoyancy field

$$
b_{0}=\tanh (x),
$$

and constant $\nu$ with $E=0.1$. The streamfunction is defined by $u=\partial \psi / \partial z$ and $w=$ $-\partial \psi / \partial x$, and in this case is

$$
\psi_{0}=-E K_{0}^{\prime}(z / \sqrt{E}) \frac{\partial b_{0}}{\partial x} .
$$

The streamfunction corresponds to anti-clockwise circulation in the $x-z$ plane and will lead to stable stratification inside the front. There is upwelling on the high buoyancy side of the front and downwelling on the low buoyancy side in regions of negative and positive curvature in the buoyancy field, respectively. The along-front velocity $\left(v_{0}\right)$ consists of jets perpendicular to the buoyancy gradient and is in the same direction as the thermal wind velocity but with reduced shear near the top and bottom boundaries.

To demonstrate the time-dependence of the leading order velocity field, Figure 3 shows $K(z, \tau)$ for $E=0.1$ and $0.01, \nu=1$ and initial condition $K(z, 0)=0$. This initial condition corresponds to no flow so the system will rapidly adjust to a balanced state. Inertial waves occur with a period of $2 \pi$, corresponding to a dimensional period of $2 \pi / f$, and are damped by viscosity on a timescale of $\tau=O\left(1 / E \lambda_{1}\right)$, corresponding to a dimensional decay rate of $\lambda_{1} \bar{\nu} / H^{2}$, where $\lambda_{1}$ is the smallest non-zero eigenvalue. For constant $\nu, \lambda_{1}=\pi^{2} \approx 10$, and inertial waves will be observed for $E \ll 0.1$. Figure 3 shows that damped inertial waves occur for $E=0.01$, while for $E=0.1$, no waves are apparent since the inertial period and damping timescale are nearly equal.

\section{2. $\mathbf{O}(\mathbf{R o})$ Buoyancy Balance}

As shown above, the leading order $(O(1))$ buoyancy, $b_{0}$, is independent of depth, $z$. However, the leading order cross-front velocity, $u_{0}$, does exhibit depth-dependence, and this shear causes the front to 'slump' over, leading to a stable stratification, a result consistent with the SML model of Young (1994). This can be seen by solving the $O(R o)$ 


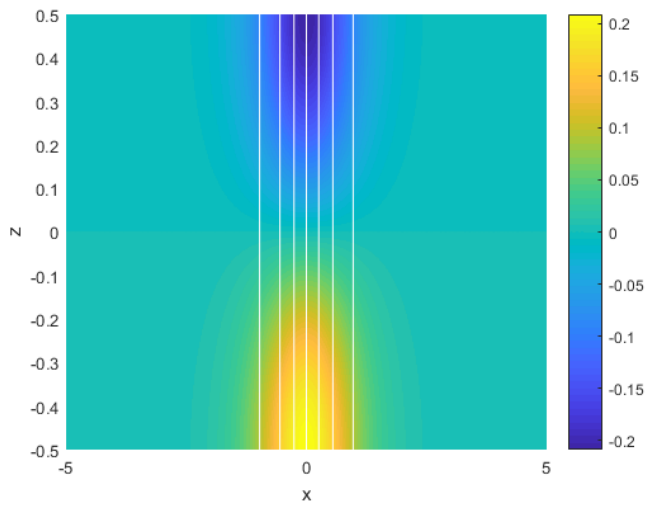

(a)

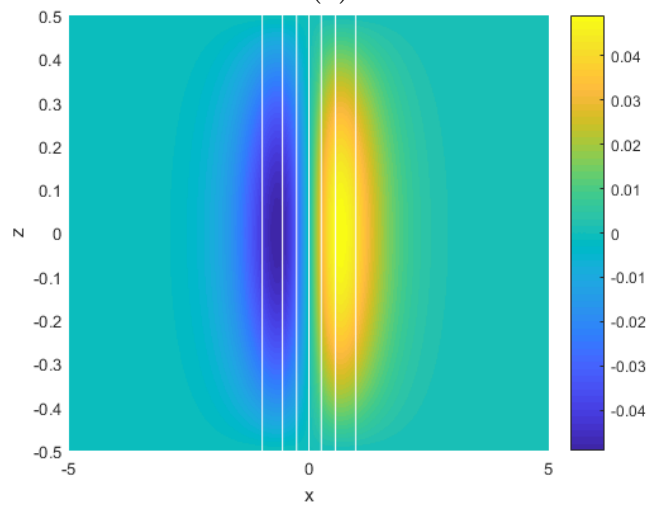

(c)

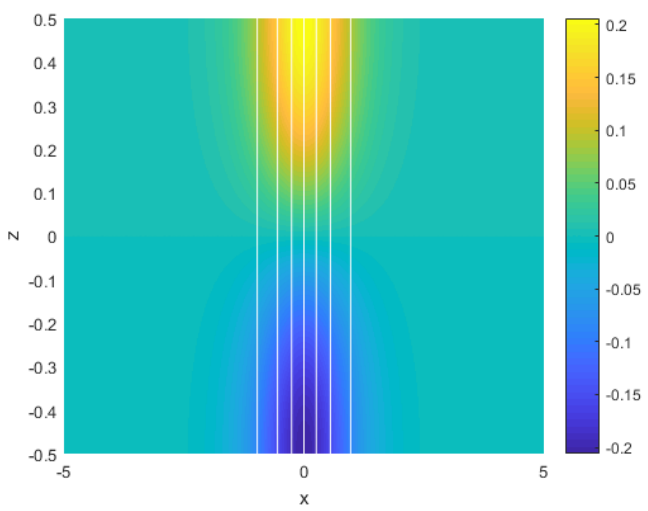

(b)

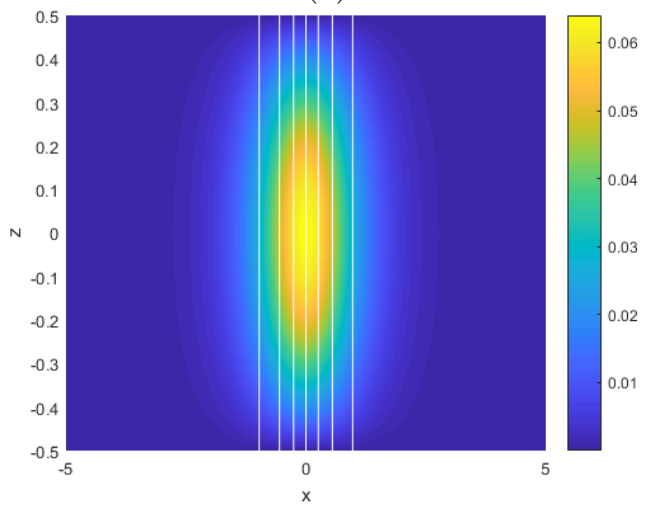

(d)

Figure 2: Leading order steady ( $\tau$ independent) velocity fields, $u_{0}(\mathrm{a}), v_{0}(\mathrm{~b}), w_{0}(\mathrm{c})$ and streamfunction, $\psi_{0}(\mathrm{~d})$, for $E=0.1, \nu=1$ and $b_{0}=\tanh x$.

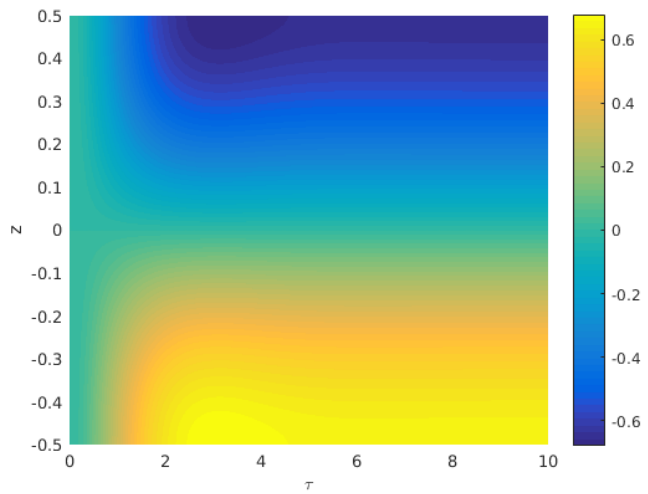

(a)

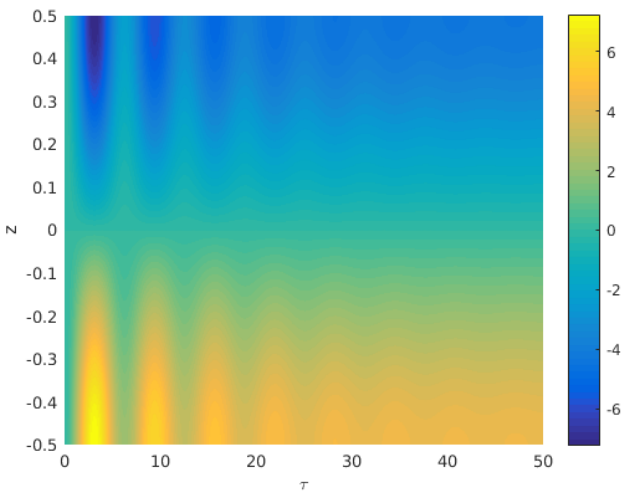

(b)

Figure 3: $K(z, \tau)$ for $E=0.1$ (a) and $0.01(\mathrm{~b})$, constant $\nu$, and initial condition $K(z, 0)=$ 0 corresponding to no initial flow. Note that the along front velocity is proportional to $K$ and the cross front velocity is proportional to $L_{\nu} K=[\partial / \partial \tau-E \partial / \partial z(\nu \partial / \partial z)] K$. 
buoyancy conservation equation, which conveniently involves just the $O(1)$ contributions to the velocity field found above.

The $O(R o)$ terms in the buoyancy conservation equation are

$$
\frac{\partial b_{1}}{\partial \tau}+u_{0} \frac{\partial b_{0}}{\partial x}+v_{0} \frac{\partial b_{0}}{\partial y}=\frac{E}{\operatorname{Pr}} \frac{\partial}{\partial z}\left(\kappa \frac{\partial b_{1}}{\partial z}\right),
$$

which can be re-written as the forced diffusion equation

$$
\left[\frac{\partial}{\partial \tau}-\frac{E}{\operatorname{Pr}} \frac{\partial}{\partial z}\left(\kappa \frac{\partial}{\partial z}\right)\right] b_{1}=-\mathbf{u}_{H 0} \cdot \nabla_{H} b_{0}
$$

where

$$
-\mathbf{u}_{H 0} \cdot \nabla_{H} b_{0}=\sqrt{E}\left(\nabla_{H} b_{0} \cdot \mathbf{K} \cdot \nabla_{H} b_{0}\right)=-\sqrt{E} L_{\nu} K\left|\nabla_{H} b_{0}\right|^{2} .
$$

It is convenient to define a function $M(z, \tau)$ such that

$$
b_{1}=-\sqrt{E} M(z, \tau)\left|\nabla_{H} b_{0}\right|^{2},
$$

and, using the definition of $L_{\nu}$, equation 3.33 becomes

$$
\left[\frac{\partial}{\partial \tau}-\frac{E}{\operatorname{Pr}} \frac{\partial}{\partial z}\left(\kappa \frac{\partial}{\partial z}\right)\right] M=\left[\frac{\partial}{\partial \tau}-E \frac{\partial}{\partial z}\left(\nu \frac{\partial}{\partial z}\right)\right] K .
$$

A general solution to equation 3.36 for $\nu \neq \kappa$ and arbitrary $\operatorname{Pr}$ is derived in Appendix B. We note that the steady state solution for $M$, denoted $M_{0}$, is given by

$$
M_{0}=\operatorname{Pr} \int_{-1 / 2}^{z}\left[\frac{\nu}{\kappa} \frac{\partial}{\partial z^{\prime}} K_{0}\left(z^{\prime} / \sqrt{E}\right)\right] d z^{\prime},
$$

which reduces to $M_{0}=\operatorname{Pr} K_{0}$ for $\nu=\kappa$. For $\operatorname{Pr}=1$ and $\nu=\kappa$ we can write equation 3.36 as

$$
\left[\frac{\partial}{\partial \tau}-E \frac{\partial}{\partial z}\left(\nu \frac{\partial}{\partial z}\right)\right](M-K)=0,
$$

so we write $M^{\prime}=M-K$ with initial condition $\left.M^{\prime}\right|_{\tau=0}=\mathcal{M}-\mathcal{K}$ corresponding to $\left.M\right|_{\tau=0}=\mathcal{M}$. We now use separation of variables to write

$$
M^{\prime}=\sum_{n=0}^{\infty} D_{n} e^{-E \lambda_{n} \tau} \mathcal{Z}_{n}(z)
$$

where

$$
D_{n}=\frac{1}{z_{n}^{2}} \int_{-1 / 2}^{1 / 2}[\mathcal{M}-\mathcal{K}] \mathcal{Z}_{n} d z
$$

hence

$$
M=K_{0}+\sum_{n=0}^{\infty}\left[B_{n}\left(\cos \tau+E \lambda_{n} \sin \tau\right)+D_{n}\right] e^{-E \lambda_{n} \tau} \mathcal{Z}_{n}(z) .
$$

The balanced buoyancy solution for $\nu=\kappa$ and arbitrary $\operatorname{Pr}$ can be written

$$
b=b_{0}(x, y, T)-\sqrt{E} \operatorname{Ro} \operatorname{Pr} K_{0}(z / \sqrt{E})\left|\nabla_{H} b_{0}\right|^{2}+O\left(R o^{2}\right) .
$$

Figure 4 shows the $O(1)$ and $O(R o)$ contributions to the buoyancy for $E=0.1, R o=0.01$ and $\operatorname{Pr}=1$ and $b_{0}(x)=\tanh (x)$. The $O(R o)$ contribution to the buoyancy results in a slumping of the front towards a stable stratification.

As the front slumps, the surface buoyancy gradient can increase corresponding to a 


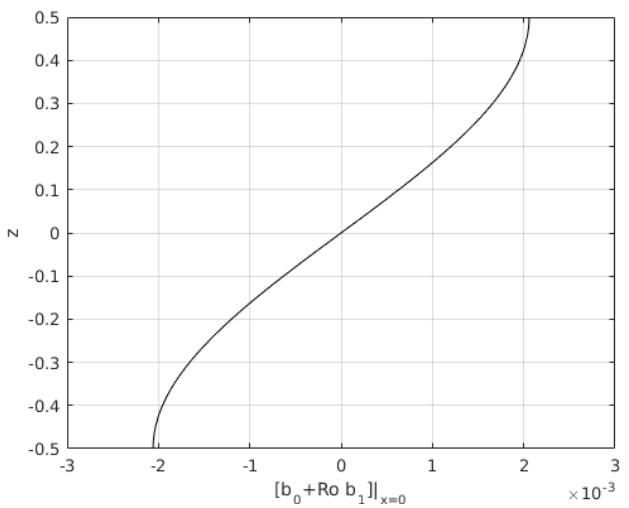

(a)



(b)

Figure 4: (a) The steady state buoyancy field to $O(R o)$ at $x=0, b(0, z)=b_{0}(0)+$ $\operatorname{Rob}_{1}(0, z)$, and (b) the $O(R o)$ correction term, $b_{1}(x, z)$. Here $E=0.1, R o=0.01$, $\operatorname{Pr}=1, \nu=\kappa=1$ and $b_{0}=\tanh (x)$.

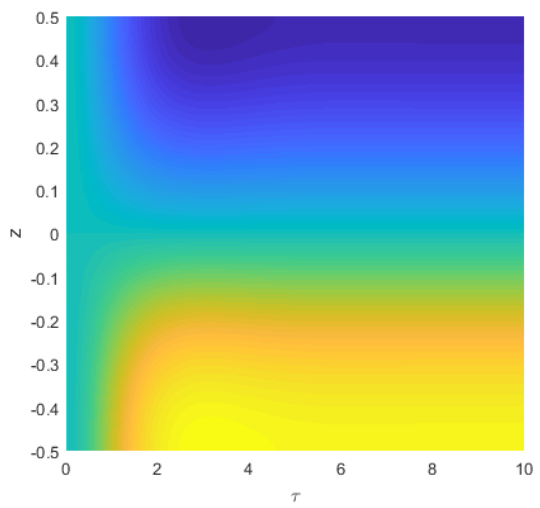

(a)

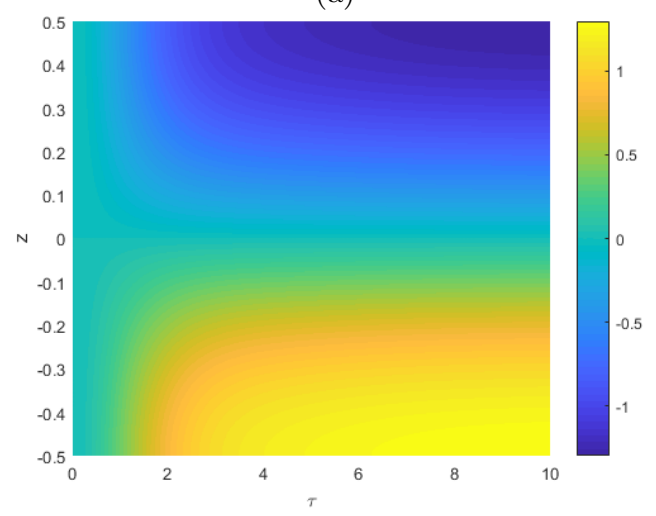

(c)

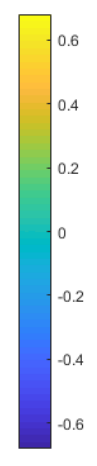

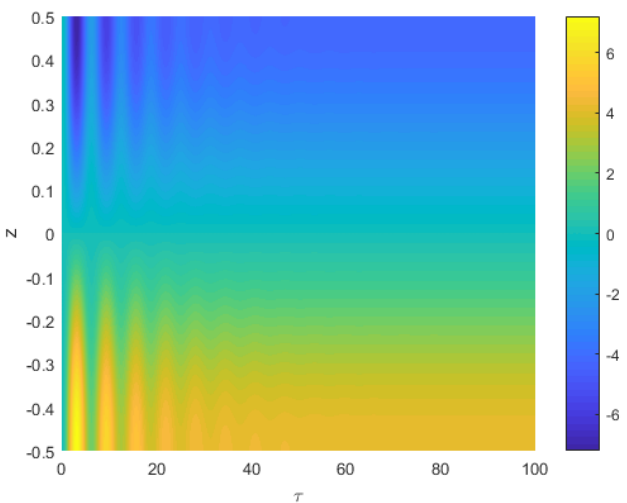

(b)



(d)

Figure 5: $M(z, \tau)$ for $(E, P r)=(0.1,1)(\mathrm{a}),(0.01,1)(\mathrm{b}),(0.1,2)(\mathrm{c})$, and $(0.01,2)(\mathrm{d})$. We use $\nu=\kappa=1$ and initial conditions $K(z, 0)=0$ corresponding to no initial flow and $M(z, 0)=0$ corresponding to an initially depth independent front. Note that $b_{1}=$ $-\sqrt{E} M\left|\nabla_{H} b_{0}\right|^{2}$. 
sharpening of the front hence allowing for short periods of frontogenesis. The horizontal buoyancy gradient is given by

$$
\nabla_{H} b=\nabla_{H} b_{0}(x, y, T)-\sqrt{E} \operatorname{Ro} \operatorname{Pr} K_{0}(z / \sqrt{E}) \nabla_{H}\left|\nabla_{H} b_{0}\right|^{2}+O\left(R o^{2}\right) .
$$

At the upper surface where $K_{0}<0$, the buoyancy gradient $\left(\left|\nabla_{H} b\right|\right)$ is larger than its initial value $\left(\left|\nabla_{H} b_{0}\right|\right)$ on the low buoyancy side of the front, while the converse is true on the high buoyancy side of the front. Along the mid-plane $(z=0) K_{0}=0$ and hence the buoyancy gradient is equal to $\nabla_{H} b_{0}$. Note that the enhancement and reduction in the buoyancy gradient relative to $\nabla_{H} b_{0}$ is $O(R o)$ and hence frontogenesis is a relatively weak effect for $R o \ll 1$.

Figure 5 shows the time dependent $M(z, \tau)$ for four pairs of values of $(E, \operatorname{Pr})$ and initial conditions of no flow and no depth dependence in the buoyancy field. We can see that the system rapidly adjusts to a balanced state with inertial waves for small $E$. Larger $\operatorname{Pr}$ results in a longer adjustment period and more stable stratification.

\section{3. $\mathbf{O}\left(\mathbf{R o}^{2}\right)$ Buoyancy Balance and Shear Dispersion}

We now consider the $O\left(R o^{2}\right)$ buoyancy balance. The depth-average of this balance allows us to determine the evolution of the leading order buoyancy field, $b_{0}$, over the slow timescale, $T$. The evolution is found to be due to both shear dispersion and horizontal diffusion, although horizontal diffusion is only important at late times.

\subsubsection{Depth-Averaged Buoyancy}

The previous two sections examined the leading order solutions to the time-dependent TTW equations. For initial conditions not in TTW balance, the leading order contributions consist of an adjustment phase with damped inertial oscillations. The evolution of $\mathbf{u}_{0}$ and $b_{1}$ during the adjustment phase involves the fast timescale, $\tau$. After the end of the adjustment phase, the front will still evolve, but now on the slow timescale, $T$. In this section, we examine the slow evolution of $b_{0}(x, z, T)$ by depth-averaging the $O\left(R o^{2}\right)$ buoyancy conservation equation.

The $O\left(R o^{2}\right)$ buoyancy equation is

$$
\frac{\partial b_{0}}{\partial T}+\frac{\partial b_{2}}{\partial \tau}+\mathbf{u}_{1} \cdot \nabla_{H} b_{0}+\mathbf{u}_{0} \cdot \nabla b_{1}=\frac{E}{\operatorname{Pr}} \frac{\partial}{\partial z}\left(\kappa \frac{\partial b_{2}}{\partial z}\right)+\frac{\epsilon^{2} E}{R o^{2} \operatorname{Pr}} \kappa \nabla_{H}^{2} b_{0}
$$

where we note that $\epsilon=O(R o)$. We define the depth average as

$$
\bar{*}=\int_{-1 / 2}^{1 / 2} * d z,
$$

and write a field $f$ as $f=\bar{f}+f^{\prime}$ where $\overline{f^{\prime}}=0$. We assume that the depth averages of the cross front components of $\mathbf{u}_{H 0}, \mathbf{u}_{H 1}, \ldots$ and the depth averages of $b_{1}, b_{2}, \ldots$ are zero. This assumption holds if the initial conditions, the viscosity, $\nu$, and the diffusivity, $\kappa$, are symmetric in $z$ about $z=0$. We note that it is possible for depth independent velocity components to develop, balanced by a depth independent pressure term, but these lead to along front velocities which do not affect buoyancy advection across the front and hence can be ignored. With this assumption, the depth-averaged buoyancy is simply

$$
\bar{b}=b_{0}(x, y, T) \text {. }
$$

We now take a depth average of equation 3.44 and note that $\bar{\kappa}=1$ to get

$$
\frac{\partial b_{0}}{\partial T}+\frac{\partial}{\partial x} \overline{u_{0} b_{1}}+\frac{\partial}{\partial y} \overline{v_{0} b_{1}}=\frac{\epsilon^{2} E}{R o^{2} P r} \nabla_{H}^{2} b_{0},
$$


where

$$
\overline{\mathbf{u}_{H 0} b_{1}}=\left(\overline{u_{0} b_{1}}, \overline{v_{0} b_{1}}\right)=E \overline{M \mathbf{K}} \cdot \nabla_{H} b_{0}\left|\nabla_{H} b_{0}\right|^{2},
$$

so we can define a second rank effective diffusivity tensor $\boldsymbol{\kappa}_{\text {eff }}$ by

$$
-\overline{\mathbf{u}_{H 0} b_{1}}=\boldsymbol{\kappa}_{e f f} \cdot \nabla_{H} b_{0},
$$

such that

$$
\boldsymbol{\kappa}_{e f f}=-E \overline{M \mathbf{K}}\left|\nabla_{H} b_{0}\right|^{2},
$$

and allowing us to write equation 3.47 as

$$
\frac{\partial b_{0}}{\partial T}=\nabla_{H} \cdot\left[\boldsymbol{\kappa}_{e f f} \cdot \nabla_{H} b_{0}+\frac{\epsilon^{2} E}{R o^{2} P r} \nabla_{H} b_{0}\right] .
$$

Since $b_{0}$ does not evolve on the short timescale $\tau$, we can describe its evolution using the steady state effective diffusivity, $\boldsymbol{\kappa}_{\text {eff }}^{(0)}$, given by

$$
\boldsymbol{\kappa}_{\text {eff }}^{(0)}=-E \overline{M_{0} \mathbf{K}_{0}}\left|\nabla_{H} b_{0}\right|^{2},
$$

where $M_{0}$ is given by equation 3.37 and

$$
\mathbf{K}_{0}=\left(\begin{array}{cc}
E \frac{\partial}{\partial z}\left(\nu \frac{\partial K_{0}}{\partial z}\right) & -K_{0} \\
K_{0} & E \frac{\partial}{\partial z}\left(\nu \frac{\partial K_{0}}{\partial z}\right)
\end{array}\right) .
$$

We note that

$$
\int_{-1 / 2}^{1 / 2} E \frac{\partial}{\partial z}\left(\nu \frac{\partial K_{0}}{\partial z}\right)\left[\int_{-1 / 2}^{z} \frac{\nu}{\kappa} \frac{\partial K_{0}}{\partial z^{\prime}} d z^{\prime}\right] d z=-\int_{-1 / 2}^{1 / 2} E \frac{\nu^{2}}{\kappa}\left(\frac{\partial K_{0}}{\partial z}\right)^{2} d z<0,
$$

hence the diagonal terms of $\overline{M_{0} \mathbf{K}_{0}}$ are negative, the diagonal terms of $\boldsymbol{\kappa}_{\text {eff }}^{(0)}$ are positive, and $\boldsymbol{\kappa}_{\text {eff }}^{(0)}$ is positive definite.

Using $\boldsymbol{\kappa}_{\text {eff }}^{(0)}$ we write equation 3.51 as

$$
\frac{\partial b_{0}}{\partial T}=\nabla_{H} \cdot\left[-E \overline{M_{0} \mathbf{K}_{0}} \cdot \nabla_{H} b_{0}\left|\nabla_{H} b_{0}\right|^{2}+\frac{\epsilon^{2} E}{R o^{2} P r} \nabla_{H} b_{0}\right]
$$

and define

$$
\mathbf{Q}(E)=-E \overline{M_{0} \mathbf{K}_{0}} / \operatorname{Pr},
$$

for positive definite matrix $\mathbf{Q}$ which depends only on $E$. Hence we can write the non-linear diffusion equation, 3.55 , as

$$
\frac{\partial b_{0}}{\partial T}=\nabla_{H} \cdot\left[\operatorname{Pr} \mathbf{Q} \cdot \nabla_{H} b_{0}\left|\nabla_{H} b_{0}\right|^{2}+\frac{\epsilon^{2} E}{R o^{2} P r} \nabla_{H} b_{0}\right] .
$$

In the case of a $y$ independent front, $b_{0}=b_{0}(x, T)$, equation 3.57 reduces to

$$
\frac{\partial b_{0}}{\partial T}=\frac{\partial}{\partial x}\left[\operatorname{Pr} Q\left(\frac{\partial b_{0}}{\partial x}\right)^{3}+\frac{\epsilon^{2} E}{R o^{2} \operatorname{Pr}} \frac{\partial b_{0}}{\partial x}\right]
$$

where

$$
Q(E)=E^{2} \int_{-1 / 2}^{1 / 2} \frac{\nu^{2}}{\kappa}\left(\frac{\partial K_{0}}{\partial z}\right)^{2} d z=E \int_{-1 / 2}^{1 / 2} \frac{\nu^{2}}{\kappa}\left(K_{0}^{\prime}\right)^{2} d z>0,
$$

since $K_{0}=K_{0}(z / \sqrt{E})$ and hence $\partial K_{0} / \partial z=E^{-1 / 2} K_{0}^{\prime}$. Equation 3.58 is the ErdoganChatwin equation, first derived for longitudinal dispersion in pipe flow by Erdogan \& 
Chatwin (1967). This equation arises in many non-rotating physical contexts where the dispersion rate is enhanced by buoyancy driven flow (Smith 1982).

\subsection{Shear Dispersion in the limit of Small Ekman Number}

Although the solution described in the previous sections is valid up to $E=O(1)$, it is useful to examine the solution for small Ekman number, specifically $E=O(R o)$. In this limit, we recover a solution obtained previously by Garrett \& Loder (1981).

We can write the buoyancy frequency, $N^{2}$, as

$$
N^{2}=\frac{\partial b}{\partial z}=R o \frac{\partial b_{1}}{\partial z}+O\left(R o^{2}\right),
$$

so

$$
N^{2}=-R o\left(\sqrt{E} \frac{\partial M}{\partial z}\right)\left|\nabla_{H} b_{0}\right|^{2}+O\left(R o^{2}\right) .
$$

During the spreading regime, $\sqrt{E} \partial M / \partial z=\sqrt{E} \partial M_{0} / \partial z=(\operatorname{Pr} \nu / \kappa) K_{0}^{\prime}$ so

$$
N^{2}=-\operatorname{Ro} \operatorname{Pr} \frac{\nu}{\kappa} K_{0}^{\prime}(z / \sqrt{E})\left|\nabla_{H} b_{0}\right|^{2}+O\left(R o^{2}\right) .
$$

In the case of $E=O(R o)$ the solution for velocity approaches that of thermal wind balance with $K_{0}(z / \sqrt{E})=-z / \sqrt{E}$ outside of thin Ekman layers near the top and bottom surfaces. These Ekman layers generate a depth independent vertical velocity in the interior by Ekman pumping (Garrett \& Loder 1981). The vertical velocity is independent of $z$ and the cross-front velocity is zero outside of the Ekman layers. Hence $K_{0}^{\prime} \rightarrow-1$ so

$$
N^{2} \sim \operatorname{Ro} \operatorname{Pr} \frac{\nu}{\kappa}\left|\nabla_{H} b_{0}\right|^{2}+O\left(R o^{2}\right)
$$

In the $2 \mathrm{~d}$ case, we have that

$$
\kappa_{e f f}=E \operatorname{Pr}\left[\overline{\frac{\nu^{2}}{\kappa}\left(K_{0}^{\prime}\right)^{2}}\right]\left|\frac{\partial b_{0}}{\partial x}\right|^{2} .
$$

and from equation 3.62 , to leading order

$$
N^{2}=-\operatorname{RoP} \operatorname{Pr} K_{0}^{\prime} \frac{\nu}{\kappa}\left|\frac{\partial b_{0}}{\partial x}\right|^{2}
$$

and hence

$$
\kappa_{e f f}=\frac{E\left[\overline{\nu^{2} K_{0}^{\prime 2} / \kappa}\right] N^{2}}{-\operatorname{Ro\nu } K_{0}^{\prime} / \kappa} .
$$

For small $E$ we have $K_{0}^{\prime} \rightarrow-1$ so $K_{0}^{\prime 2} \rightarrow 1$. If we assume $\nu$ and $\kappa$ are approximately constant and equal in the interior region, away from the Ekman layers, the effective diffusivity is

$$
\kappa_{e f f} \sim \frac{N^{2}}{R o} E
$$

in the small $E$ and Ro limit. We note that $N^{2}$ is approximately constant in the interior. Dimensionally this result corresponds to

$$
\kappa_{e f f} \sim \frac{\mathcal{N}^{2}}{f^{2}} \bar{\nu}_{d i m},
$$

where $\bar{\nu}_{\text {dim }}$ is the depth-averaged turbulent viscosity and $\mathcal{N}$ is the dimensional buoyancy frequency. This result was obtained previously by Garrett \& Loder (1981). 


\subsection{Self-Similar Solution}

As the front spreads through shear dispersion on the slow timescale, $T$, it adopts a self-similar cross-front profile. The self-similar solution can be obtained from the ErdoganChatwin equation (Eq. 3.58). In this section we will consider a two-dimensional front with $b_{0}=F(x, T)$. This assumption isn't necessary for the analysis but simplifies the notation. In this case, equation 3.58 can be written

$$
\frac{\partial F}{\partial T}=\frac{\partial}{\partial x}\left[\left(c_{0}+c_{2}\left(\frac{\partial F}{\partial x}\right)^{2}\right) \frac{\partial F}{\partial x}\right],
$$

where

$$
c_{0}=\frac{\epsilon^{2} E}{R o^{2} P r} \quad \text { and } \quad c_{2}=\operatorname{Pr} Q(E),
$$

subject to boundary and initial conditions

$$
F \rightarrow \pm 1 \quad \text { as } \quad x \rightarrow \pm \infty \quad \text { and } \quad F(x, 0)=f(x) .
$$

We now consider the limit in which we can neglect horizontal diffusion, i.e. $c_{0} \simeq 0$. This limit gives

$$
\frac{\partial F}{\partial T}=c_{2} \frac{\partial}{\partial x}\left(\frac{\partial F}{\partial x}\right)^{3}
$$

We now seek a similarity solution of the form

$$
F(x, T)=F(\eta) \quad \text { for similarity variable } \quad \eta=\frac{x}{\gamma T^{\alpha}} .
$$

We note that it will not in general be possible to satisfy the initial condition $F(x, 0)=$ $f(x)$, however Smith (1982) showed that, in the case where the non-linear diffusion dominates, the solutions to equation 3.69 will limit to this similarity solution for all nearby initial conditions. Using equation 3.72 , we have

$$
-\frac{\alpha \eta}{T} F^{\prime}=\frac{c_{2}}{\gamma^{4} T^{4 \alpha}}\left(F^{\prime 3}\right)^{\prime} .
$$

We let $\alpha=1 / 4$ and $k_{2}=4 c_{2} / \gamma^{4}$ so equation 3.74 becomes

$$
\eta F^{\prime}=-k_{2}\left(F^{\prime 3}\right)^{\prime}
$$

which we can solve for $F^{\prime}$, without loss of generality we assume that $F^{\prime}(0)=1$. The solution for $F^{\prime}$ is

$$
F^{\prime}(\eta)= \begin{cases}0, & \eta<-\sqrt{3 k_{2}} \\ \sqrt{1-\frac{\eta^{2}}{3 k_{2}}}, & \eta \in\left[-\sqrt{3 k_{2}}, \sqrt{3 k_{2}}\right], \\ 0, & \eta>\sqrt{3 k_{2}}\end{cases}
$$

which we integrate to obtain the solution (Smith 1982)

$$
F(\eta)= \begin{cases}-1, & \eta<-\sqrt{3 k_{2}} \\ \frac{1}{2}\left[\eta \sqrt{1-\frac{\eta^{2}}{3 k_{2}}}+\sqrt{3 k_{2}} \arcsin \left(\frac{\eta}{\sqrt{3 k_{2}}}\right)\right], & \eta \in\left[-\sqrt{3 k_{2}}, \sqrt{3 k_{2}}\right] . \\ 1, & \eta>\sqrt{3 k_{2}}\end{cases}
$$

By matching $F\left( \pm \sqrt{3 k_{2}}\right)= \pm 1$ we obtain $\sqrt{3 k_{2}}=4 / \pi$ hence

$$
k_{2}=\frac{16}{3 \pi^{2}},
$$




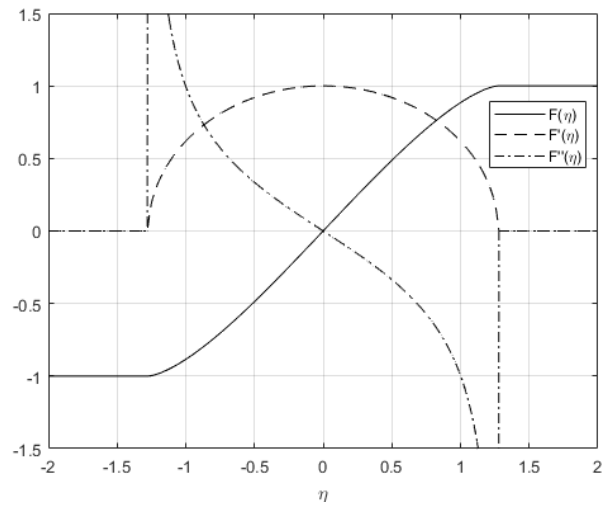

(a)

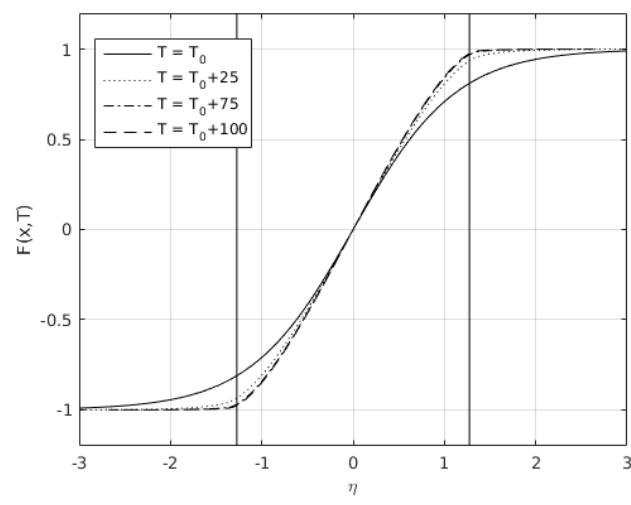

(b)

Figure 6: (a) Plots of the analytic similarity solution, $F(\eta)$, to equation 3.72 and derivatives $F^{\prime}(\eta)$ and $F^{\prime \prime}(\eta)$. Note that $b_{0} \propto F,\left(u_{0}, v_{0}\right) \propto F^{\prime}$ and $w_{0} \propto F^{\prime \prime}$. (b) The numerical solution to equation $3.69, F(x, T)$, with initial condition $b\left(x, T_{0}\right)=\tanh (x)$ for $T_{0}=4, E=0.1, \nu=\kappa=1, R o=0.05, \epsilon=0.002$ and $\operatorname{Pr}=1$ corresponding to $c_{0}=1.6 \times 10^{-4}$ and $c_{2}=0.0208$. The initial condition in the numerical solution is imposed at $T=T_{0}$ to avoid the singularity in $\eta$ at $T=0$. The vertical lines in (b) show the predicted frontal edges, $\eta= \pm 4 / \pi$.

and

$$
\gamma=\left(\frac{3 \pi^{2} c_{2}}{4}\right)^{1 / 4} .
$$

As $k_{2}$ is independent of $E$ and $\operatorname{Pr}$ we can see that the form of the solution is the same for all parameter ranges with the parameters entering only through the spreading parameter $\gamma$ which describes the frontal width.

We note that $F$ and $F^{\prime}$ are continuous while $F^{\prime \prime}$ diverges at the edge of the front, i.e. as $\eta \rightarrow \pm \sqrt{3 k_{2}}$, as

$$
F^{\prime \prime}(\eta)= \begin{cases}0, & \eta<-\sqrt{3 k_{2}} \\ -\frac{\eta}{3 k_{2} \sqrt{1-\eta^{2} / 3 k_{2}}}, & \eta \in\left(-\sqrt{3 k_{2}}, \sqrt{3 k_{2}}\right) . \\ 0, & \eta>\sqrt{3 k_{2}}\end{cases}
$$

When horizontal diffusion is included as in Eq. 3.69, this singularity is smoothed.

Figure 6.a shows the similarity solution, $F(\eta)$, and its first and second derivative. The function $F(\eta)$ is approximately linear inside the front with regions of large curvature at the edges. Figure 6.b shows the numerical solution to equation 3.69 for $c_{0}=1.6 \times 10^{-4}$ and $c_{2}=0.0208$ at $T=T_{0}, T=T_{0}+25, T=T_{0}+75$ and $T=T_{0}+100$. The initial profile is given by $b\left(x, T_{0}\right)=\tanh (x)$ for $T_{0}=4$ and we see that this profile has evolved towards the similarity solution, $F(\eta)$, by time $T=T_{0}+75$ before spreading out in a self similar manner for larger $T$.

By considering the relative magnitudes of the shear dispersion and horizontal diffusion terms, we find that our similarity solution is valid for

$$
t \ll \frac{\operatorname{Ro}^{3} \operatorname{Pr}^{3} Q(E)}{\epsilon^{4} E^{2}} .
$$

Figure 7 shows logarithmic plots of $Q$ and $\gamma / \operatorname{Pr}^{1 / 4}$ as functions of Ekman number for 


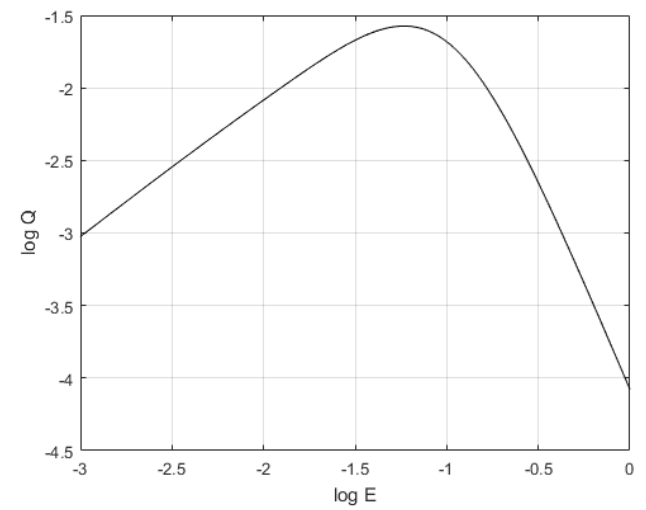

(a)

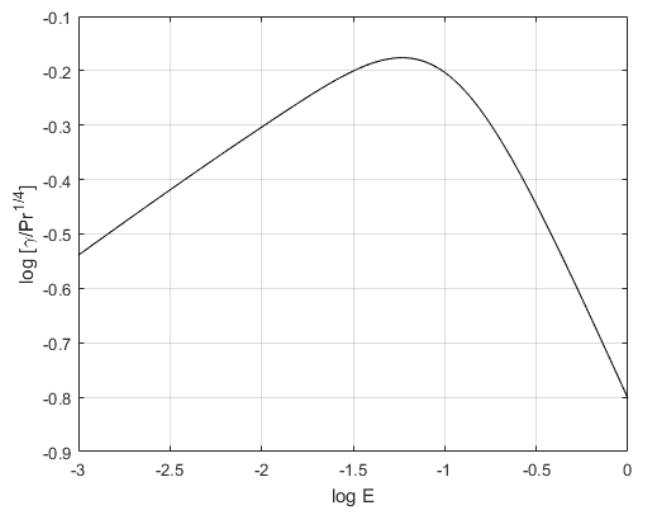

(b)

Figure 7: Logarithmic plots of $Q(E)$ (a) and $\gamma / \operatorname{Pr}^{1 / 4}$ (b) as functions of $E$ for constant $\nu$. Note that $\gamma / \operatorname{Pr}^{1 / 4}$ depends only on $E$ and the frontal width is proportional to $\gamma T^{1 / 4}$.

constant $\nu$. Note from Eq. 3.69 and 3.70 that the effective diffusivity is proportional to $\operatorname{Pr} Q$, while the frontal width is given by

$$
l_{f}(T)=8 \gamma T^{1 / 4} / \pi,
$$

since the edges of the front correspond to $\eta= \pm \sqrt{3 k_{2}}$. As described in $\S 3.3 .1$ the effective diffusivity depends on the departure from the depth-averaged buoyancy, $b_{1}=$ $-\sqrt{E} \operatorname{Pr} K_{0}\left|\partial b_{0} / \partial x\right|^{2}$, and the cross-front velocity, $u_{0}=-\sqrt{E} K_{0}^{\prime \prime} \partial b_{0} / \partial x$.

In the limit of small $E$, the cross-front velocity becomes small as the system is approximately in thermal wind balance. As discussed in $\S 3.4$ this leads to a small effective diffusivity, consistent with the result that $Q \sim E$ and $\gamma \sim E^{1 / 4}$ in this limit (see Figure 7 ). In the limit of large $E$, strong vertical mixing causes the stratification and the crossfront velocity to decrease with increasing $E$ resulting in a smaller effective diffusivity and slower spreading. In the limit of large $E, Q \sim E^{-3}$ and $\gamma \sim E^{-3 / 4}$, consistent with this description. The effective diffusivity and spreading rate are maximum for an intermediate value of $E \approx 0.06$. See Appendix A for a more complete description of the dependence of $u_{0}$ and $b_{1}$ on $E$.

Ferrari \& Young (1997) consider a frontal problem using an 'intermittent mixing' parametrisation where the tracers are advected freely until time $t=t_{m i x}$ where the vertical tracer profile is instantaneously set to its vertical average and the process repeated. The governing equation for the depth independent buoyancy profile is of the same form as equation 3.72 with a different value of $c_{2}$ hence admits the same similarity solution. This will be true for any parametrised model in which the leading order velocity is linear in the buoyancy gradient, $u \approx c(z) \bar{b}_{x}$, and the leading order buoyancy flux scales as $F_{b}=\overline{u b} \sim \overline{u D_{z} u} \bar{b}_{x} \sim \bar{b}_{x}^{3}$ for some linear, $z$ dependent, operator, $D_{z}$, which is determined by the parametrisation. We note that the vertical profiles of velocity and buoyancy differ between models though the leading order depth-averaged tracer profiles have the same form over long times.

Figure 8 shows the long term evolution of the background buoyancy, buoyancy gradients and cross front velocity using the similarity solution. We can see that as the front spreads, both the vertical and horizontal buoyancy gradients decrease and the cross front velocity decelerates. Since the vertical buoyancy stratification formed by the initial slumping is maintained by the cross front velocity shear, this stratification weakens as 




(a)

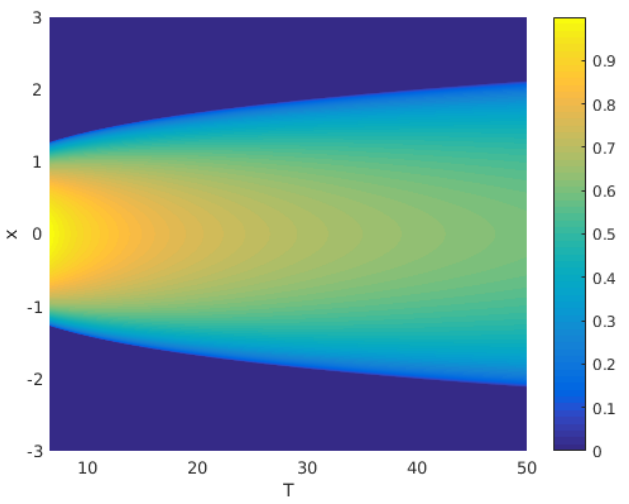

(c)

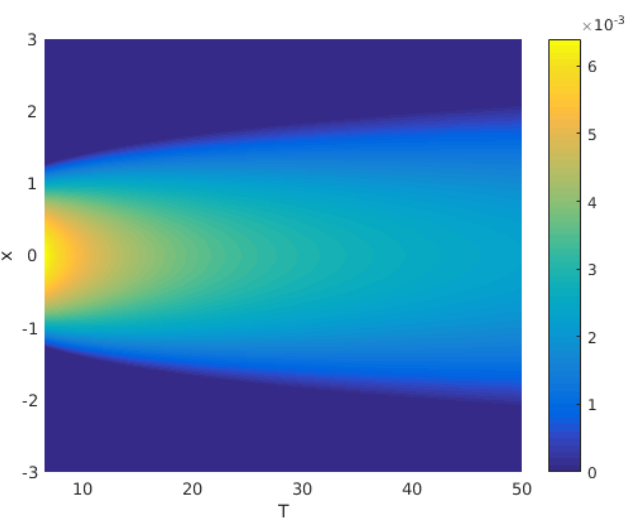

(b)

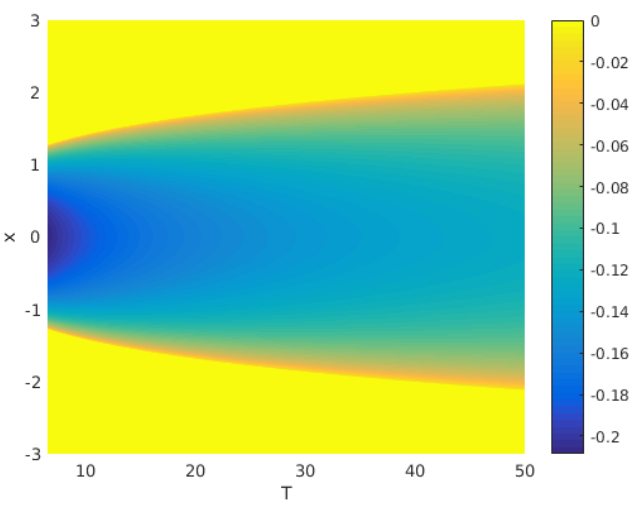

(d)

Figure 8: Plots of $b_{0}(x, T)(\mathrm{a}), N_{0}^{2}(x, z, T)=R o \partial b_{1} / \partial z$ at $z=0(\mathrm{~b}), M_{0}^{2}(x, T)=\partial b_{0} / \partial x$ (c) and $u_{0}(x, z, T)$ at $z=1 / 2(\mathrm{~d})$ for $E=0.1, R o=0.01$ and $\operatorname{Pr}=1$. We use the similarity solution for $b_{0}$ and assume that all transients have decayed. Note that $b_{0} \sim F$, $N_{0}^{2} \sim F^{\prime 2}$ and $M_{0}^{2}, u_{0} \sim F^{\prime}$.

expected when the cross front velocity decelerates. The along front velocity, $v_{0}$, and vertical velocity, $w_{0}$, also decrease as the front spreads.

Note that the vertical structure of the buoyancy perturbation, $b_{1}$, and the velocities, $\left(u_{0}, v_{0}, w_{0}\right)$, are independent of the long timescale, $T$, and assuming that any transients have decayed, only depend on depth $z$, and Ekman number.

\subsection{Summary of analytic solution}

Combining our results from the $O(1), O(R o)$ and $O\left(R o^{2}\right)$ balances, we can write our analytic solution as

$$
\begin{aligned}
\mathbf{u}_{H}(x, y, z, \tau, T) & =-\sqrt{E} \mathbf{K}(z, \tau) \cdot \nabla_{H} b_{0}(x, y, T)+O(R o) \\
w(x, y, z, \tau, T) & =E\left[\int_{-1 / 2}^{z} \frac{-L_{\nu} K\left(z^{\prime}, \tau\right)}{\sqrt{E}} d z^{\prime}\right] \nabla_{H}^{2} b_{0}(x, y, T)+O(R o) \\
b(x, y, z, \tau, T) & =b_{0}(x, y, T)-\operatorname{Ro} \sqrt{E} M(z, \tau)\left|\nabla_{H} b_{0}(x, y, T)\right|^{2}+O\left(R o^{2}\right),
\end{aligned}
$$


where $b_{0}$ satisfies

$$
\frac{\partial b_{0}}{\partial T}=\nabla_{H} \cdot\left[\operatorname{Pr} \mathbf{Q} \cdot \nabla_{H} b_{0}\left|\nabla_{H} b_{0}\right|^{2}+\frac{\epsilon^{2} E}{R o^{2} P r} \nabla_{H} b_{0}\right] .
$$

\section{Conclusions and Discussion}

Here, we examined the influence of small-scale turbulence on the adjustment and spreading of density fronts in a rotating reference frame using a multiple time-scale analysis and asymptotic analysis. After a short initial adjustment period, a self-similar spreading regime develops in which an effective horizontal diffusion results from the coupling of a vertically-sheared cross-front flow and vertical diffusion. During the initial adjustment period, weak frontogenesis is possible due to the slumping of front while the long-term spreading is always frontolytic.

The time dependence and similarity solutions during the spreading phase have been calculated analytically using an expansion in small Rossby number, allow us to predict the rate of spreading for a wide range of Ekman and Rossby numbers. The dominant balance in the buoyancy equation is between vertical diffusion and cross-front advection, resulting in a buoyancy field consisting of both spreading and slumping components in a dynamic equilibrium. The dominant balance in the momentum equations is the TTW balance between Coriolis forces, vertical momentum mixing and horizontal pressure gradients for which analytic and numerical solutions have been presented. This balance holds until the effects of horizontal diffusion becomes significant at late times.

Horizontal spreading of the depth-averaged buoyancy results from shear dispersion, which dominates the horizontal turbulent diffusion in the frontal region until late times (equation 3.81). The spreading is described by the non-linear Erdogan-Chatwin (Erdogan \& Chatwin 1967) equation which arises in many contexts and has solutions which tend to a similarity solution in the region where shear dispersion dominates. Shear dispersion leads to a cross-front density profile that is nearly linear inside the front with high curvature at the edges of the frontal zone. High curvature is associated with large vertical velocity through the TTW balance which results in up/down-welling confined to thin bands at the edges of the front. Qualitatively this result is similar to the velocity confinement effect observed by Shakespeare \& Taylor (2013) for inviscid strain-driven fronts though it arises from a different mechanism.

Dimensionally, we can write the frontal width as

$$
L_{f}(t)=\left(\frac{3072}{\pi^{2}}\right)^{1 / 4}\left[\frac{Q(E) H^{2} \Delta b^{2} P r}{f^{3}}\right]^{1 / 4} t^{1 / 4},
$$

using equation 3.82 and hence the spreading rate is given by

$$
\frac{d L_{f}}{d t}=\frac{768}{\pi^{2}}\left[\frac{Q(E) H^{2} \Delta b^{2} P r}{f^{3}}\right] L_{f}^{-3},
$$

where $Q(E)$ is defined in equation 3.59. For a typical ocean front with $H \sim 100 \mathrm{~m}$, $L_{f} \sim 10 \mathrm{~km}, f \sim 10^{-4} \mathrm{~s}^{-1}$ and $\Delta \rho \sim 0.1 \mathrm{~kg} \mathrm{~m}^{-3}$, the observed frontal spreading would occur at a rate of order $1 \mathrm{~km}$ per day for an Ekman number of $E=0.1$ (corresponding to a turbulent viscosity of $\nu \sim 0.1 \mathrm{~m}^{2} \mathrm{~s}^{-1}$ ). By comparison, spreading due to horizontal diffusion would just be on the order of $1 \mathrm{~m}$ per day for the same parameters.

Using scaling arguments, we can relate the Ekman number to the turbulent viscosity associated with convection driven by a surface heat flux, $J_{0}$, or a surface wind stress, $\tau_{w}$. In the case of convection, the turbulent Ekman number scales according to (Taylor \& 
Ferrari 2011)

$$
E \simeq \frac{C}{f}\left(\frac{J_{0} \alpha g}{c_{p} \rho_{0} H^{2}}\right)^{1 / 3}
$$

where $H$ is the depth of the convective layer, $\rho_{0}$ is the water density, $c_{p}$ is the heat capacity, $\alpha$ is the thermal expansion coefficient, $C$ is an empirical scaling constant and $g$ is gravitational acceleration. Using typical values for the ocean including a convective layer depth of $100 \mathrm{~m}$ and $f=10^{-4} \mathrm{~s}^{-1}$, typical heat flux values in the range $Q_{0}=1-1000 \mathrm{~W} / \mathrm{m}^{2}$ correspond to Ekman numbers ranging from $E \simeq 0.1-1$. Similarly, the Ekman number can be related to the wind stress using (Enriquez \& Taylor 2015)

$$
E \simeq \frac{C}{f H}\left(\frac{\tau_{w}}{\rho_{0}}\right)^{1 / 2}
$$

and a wind stress of $\tau_{w}=1 \mathrm{Nm}^{-2}$ corresponds to $E \simeq 0.1$.

In our analysis, the only mechanism driving frontogenesis is the coupling between the cross-front velocity and the along-front thermal wind through the turbulent mixing. For less idealised flows, the tendency for fronts to sharpen or spread is governed by a competition between the classical frontogenesis function and the effects of turbulent mixing. Interestingly we observe that turbulent mixing can both sharpen and spread fronts with sharpening associated with frontogenesis during an initial adjustment phase and spreading associated with shear dispersion.

In the ocean and atmosphere, deformation of fronts by larger scale circulation can generate cross-front circulation that can drive further frontogenesis (Hoskins \& Bretherton 1972). Shear dispersion through vertical mixing might provide a mechanism to arrest deformation-driven frontogenesis, which can lead to finite-time singularities (Hoskins \& Bretherton 1972). Future work could include externally imposed large-scale strain to examine frontal arrest, which would provide insight into the properties of equilibrated fronts.

To simplify our analysis, we have kept the viscosity and diffusivity independent of time. Recent observations and numerical simulations have found that turbulence can be significantly enhanced at strong fronts including the Kuroshio (D'Asaro et al. 2011), Gulf Stream (Thomas et al. 2013) and the California Current (Johnston et al. 2011). On the other hand, the stable stratification that develops as a front slumps has been implicated in the suppression of turbulent mixing (Taylor 2016; Taylor \& Ferrari 2010). It remains unclear how small-scale turbulence depends on the large-scale properties of the front and the surface wind and buoyancy forcing. In either case, the turbulent viscosity and diffusivity likely vary with position across the front and in time, effects which are neglected here. Varying levels of small-scale turbulence could lead to a feedback between the evolution of the front through TTW balance and turbulent mixing. The coupled response of turbulence and frontal dynamics will be the topic of future work.

The authors would like to thank Raffaele Ferrari, Jim McWilliams, Leif Thomas, and Jacob Wenegrat for stimulating and helpful conversations. We are also very grateful to Bill Young for comments on an early draft of the paper and for pointing out various connections with previous literature on shear dispersion. 


\section{Appendix A. Analytic Solution for the $\mathrm{O}(1)$ Velocity for Constant $\nu$}

We assume constant viscosity, $\nu=1$, to find an analytic solution for the structure function $K_{0}$. Equation 3.15 becomes

$$
-K_{0}^{(4)}=K_{0}+\zeta
$$

with boundary conditions $K_{0}^{\prime}=K_{0}^{\prime \prime \prime}=0$ at $\zeta= \pm \zeta_{0}$ where $\zeta_{0}=1 / \sqrt{4 E}$. This is a fourth order ODE which can be solved analytically. The exponents of the independent solutions are $(1 \pm i) / \sqrt{2}$ and $(-1 \pm i) / \sqrt{2}$ so, since the system is clearly odd in $\zeta$, we use symmetry to obtain the solution

$$
K_{0}=-\zeta+C_{+} \operatorname{cs}(\zeta)+C_{-} \operatorname{sc}(\zeta)
$$

where

$$
\begin{aligned}
& \operatorname{cc}(\zeta)=\cosh (\zeta / \sqrt{2}) \cos (\zeta / \sqrt{2}), \\
& \operatorname{cs}(\zeta)=\cosh (\zeta / \sqrt{2}) \sin (\zeta / \sqrt{2}), \\
& \operatorname{ss}(\zeta)=\sinh (\zeta / \sqrt{2}) \sin (\zeta / \sqrt{2}), \\
& \operatorname{sc}(\zeta)=\sinh (\zeta / \sqrt{2}) \cos (\zeta / \sqrt{2}) .
\end{aligned}
$$

From the boundary conditions, $K_{0}^{\prime}\left(\zeta_{0}\right)=K_{0}^{\prime \prime \prime}\left(\zeta_{0}\right)=0$, we get

$$
\left(\begin{array}{cc}
\operatorname{cc}\left(\zeta_{0}\right)+\operatorname{ss}\left(\zeta_{0}\right) & \operatorname{cc}\left(\zeta_{0}\right)-\operatorname{ss}\left(\zeta_{0}\right) \\
\operatorname{cc}\left(\zeta_{0}\right)-\operatorname{ss}\left(\zeta_{0}\right) & -\operatorname{cc}\left(\zeta_{0}\right)-\operatorname{ss}\left(\zeta_{0}\right)
\end{array}\right)\left(\begin{array}{l}
C_{+} \\
C_{-}
\end{array}\right)=\left(\begin{array}{c}
\sqrt{2} \\
0
\end{array}\right),
$$

which we invert to get

$$
C_{ \pm}=\frac{1}{\sqrt{2}} \frac{\operatorname{cc}\left(\zeta_{0}\right) \pm \operatorname{ss}\left(\zeta_{0}\right)}{\operatorname{cc}^{2}\left(\zeta_{0}\right)+\operatorname{ss}^{2}\left(\zeta_{0}\right)} .
$$

We can also solve for $\mathcal{Z}_{n}$ analytically using equation 3.21 which reduces to

$$
\frac{\partial^{2} \mathcal{Z}_{n}}{\partial z^{2}}=-\lambda_{n} \mathcal{Z}_{n}
$$

We obtain solutions

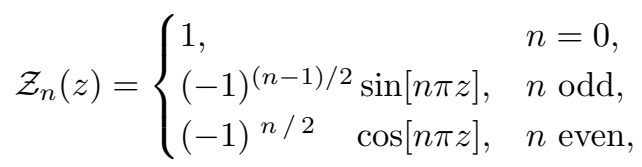

for

$$
\lambda_{n}=n^{2} \pi^{2}
$$

and

$$
z_{n}^{2}= \begin{cases}1, & n=0 \\ \frac{1}{2}, & n \in \mathbb{N}\end{cases}
$$

and integrate to get



We plot $K_{0}$ and $\mathcal{Z}_{n}$ in Appendix $\mathrm{C}$ when considering the numerical solutions for general $\nu$, see figures 12.a, 12.b and 11 .

We now consider the Ekman number dependence of $K_{0}$ in the case of constant $\nu$ and $\kappa$ and $\operatorname{Pr}=1$. We find that there are regimes for small and large $E$ where the maximum 


$\begin{array}{ccccccc}\text { Function } & K_{0}(\zeta) & K_{0}^{\prime}(\zeta) & K_{0}^{\prime \prime}(\zeta) & \sqrt{E} K_{0}(\zeta) & E K_{0}^{\prime}(\zeta) & \sqrt{E} K_{0}^{\prime \prime}(\zeta) \\ \text { Small } E & E^{-1 / 2} & 1 & 1 & 1 & E & E^{1 / 2} \\ \text { Large } E & E^{-5 / 2} & E^{-2} & E^{-3 / 2} & E^{-2} & E^{-1} & E^{-1}\end{array}$

Table 2: The Ekman number dependence of the maximum values of $K_{0}(\zeta), K_{0}^{\prime}(\zeta), K_{0}^{\prime \prime}(\zeta)$, $\sqrt{E} K_{0}(\zeta), E K_{0}^{\prime}(\zeta)$ and $\sqrt{E} K_{0}^{\prime \prime}(\zeta)$ for $\zeta=z / \sqrt{E}$ in the case of small and large $E$. Here we ignore any constant coefficients.

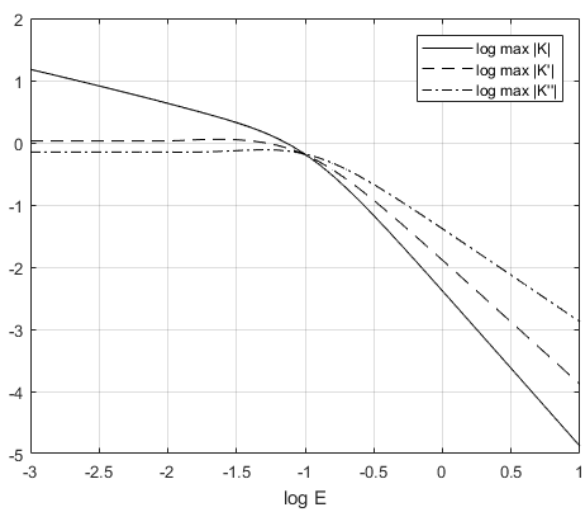

(a)

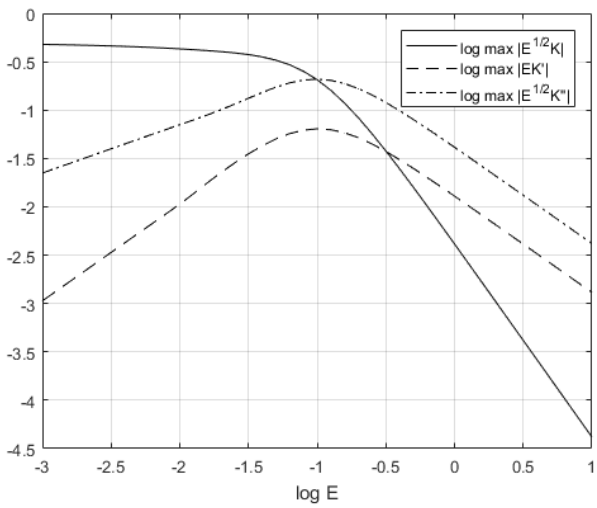

(b)

Figure 9: Logarithmic plots of the maximum magnitude as a function of Ekman number of $(\mathrm{a}): K_{0}(\zeta), K_{0}^{\prime}(\zeta)$ and $K_{0}^{\prime \prime}(\zeta)$ and $(\mathrm{b}): \sqrt{E} K_{0}(\zeta), E K_{0}^{\prime}(\zeta)$ and $\sqrt{E} K_{0}^{\prime \prime}(\zeta)$ for $\zeta=z / \sqrt{E}$.

values of $K_{0}$ and its derivatives obey power law dependences on $E$. These are given in table 2 and can be calculated by expanding equation A 2 for small and large $E$.

The maximum values for $K_{0}$ and $K_{0}^{\prime \prime}$ occur at the boundaries as the horizontal velocity is highest at the surface while the maximum for $K_{0}^{\prime}$ occurs in the interior of the vertical domain corresponding to the location of the maximum vertical velocity.

Figure 9 shows logarithmic plots of the maximum magnitude of $K_{0}(\zeta), K_{0}^{\prime}(\zeta), K_{0}^{\prime \prime}(\zeta)$, $\sqrt{E} K_{0}(\zeta), E K_{0}^{\prime}(\zeta)$ and $\sqrt{E} K_{0}^{\prime \prime}(\zeta)$ as functions of Ekman number. We can see that for small $E$ the along-front velocity, proportional to $\sqrt{E} K_{0}$, approaches a constant value corresponding to the surface velocity from the Thermal Wind balance profile. The crossfront and vertical velocities tend to zero for small $E$ in agreement with Thermal Wind balance. For large $E$ all velocity components become small as motion is damped by the high viscosity. We note that there is a maximum for both the vertical and cross-front velocities at $E \approx 0.1$. Analytically we can show that

$$
K_{0}^{\prime} \sim \begin{cases}-1, & E \ll 1, \\ \frac{1}{E^{2}}\left[\frac{z^{2}-1 / 4}{16}-\frac{z^{4}-1 / 16}{24}\right], & E \gg 1,\end{cases}
$$

hence

$$
Q(E) \sim \begin{cases}E, & E \ll 1, \\ \frac{31}{362880} E^{-3}, & E \gg 1,\end{cases}
$$

in agreement with figure 7 . 


\section{Appendix B. Solution for $M$ with arbitrary $\nu$ and $\kappa$}

To solve for $M$ with arbitrary $\nu$ and $\kappa$, we take the Laplace transform of equation 3.36 to get

$$
\left[s-\frac{E}{\operatorname{Pr}} \frac{\partial}{\partial z}\left(\kappa \frac{\partial}{\partial z}\right)\right] \widetilde{M}(z, s)-M(z, 0)=\widetilde{P}(z, s),
$$

where we take $M(z, 0)=\mathcal{M}(z)$ as our initial state. We usually take $\mathcal{M}=0$ corresponding to a depth independent front, if we were instead to consider a balanced initial setup, the solution would be given by the steady state solution, $M_{0}$. The forcing term, $\widetilde{P}$ is given by

$$
\widetilde{P}(z, s)=\left[s-E \frac{\partial}{\partial z}\left(\nu \frac{\partial}{\partial z}\right)\right] \widetilde{K}(z, s)-\mathcal{K}(z),
$$

where we can use equation 3.25 to write

$$
\widetilde{K}(z, s)=\frac{1}{s} K_{0}(z / \sqrt{E})+\sum_{n=0}^{\infty} B_{n}\left[\frac{s+2 E \lambda_{n}}{\left(s+E \lambda_{n}\right)^{2}+1}\right] \mathcal{Z}_{n}(z),
$$

SO

$$
\widetilde{P}(z, s)=\left[K_{0}-\mathcal{K}\right]-\frac{1}{s} E \frac{\partial}{\partial z}\left(\nu \frac{\partial K_{0}}{\partial z}\right)+\sum_{n=0}^{\infty} B_{n}\left[\frac{\left(s+E \lambda_{n}\right)\left(s+2 E \lambda_{n}\right)}{\left(s+E \lambda_{n}\right)^{2}+1}\right] \mathcal{Z}_{n}(z) .
$$

We note that

$$
K_{0}-\mathcal{K}=-\sum_{n=0}^{\infty} B_{n} \mathcal{Z}_{n}(z)
$$

hence

$$
\widetilde{P}(z, s)=-\frac{1}{s} E \frac{\partial}{\partial z}\left(\nu \frac{\partial K_{0}}{\partial z}\right)+\sum_{n=0}^{\infty} B_{n}\left[\frac{E \lambda_{n}\left(s+E \lambda_{n}\right)-1}{\left(s+E \lambda_{n}\right)^{2}+1}\right] \mathcal{Z}_{n}(z) .
$$

We now write

$$
\widetilde{M}=\sum_{n=0}^{\infty} \widetilde{C}_{n}(s) \mathcal{Y}_{n}(z)
$$

where the eigenfunctions, $\mathcal{Y}_{n}$, are given by

$$
\frac{\partial}{\partial z}\left(\kappa \frac{\partial \mathcal{Y}_{n}}{\partial z}\right)=-\mu_{n} \mathcal{Y}_{n}
$$

for eigenvalues $\mu_{n}$ where $n=0,1,2, \ldots$ We also define the orthogonality relation

$$
\int_{-1 / 2}^{1 / 2} \mathcal{Y}_{n} \mathcal{Y}_{m} d z=y_{n}^{2} \delta_{n m}
$$

for constant $y_{n}$ representing the normalisation of $\mathcal{Y}_{n}$.

We can expand $\widetilde{P}$ and $\mathcal{M}$ in the basis $\mathcal{Y}_{n}$ so equation B 1 becomes

$$
\sum_{n=0}^{\infty}\left(s+\frac{E}{P r} \mu_{n}\right) \widetilde{C}_{n}(s) \mathcal{Y}_{n}(z)=\sum_{n=0}^{\infty}\left(M_{n}+\widetilde{E}_{n}(s)\right) \mathcal{Y}_{n}(z),
$$

where

$$
\widetilde{E}_{n}(s)=\frac{1}{y_{n}^{2}} \int_{-1 / 2}^{1 / 2} \widetilde{P}(z, s) \mathcal{Y}_{n}(z) d z
$$


and

$$
M_{n}=\frac{1}{y_{n}^{2}} \int_{-1 / 2}^{1 / 2} \mathcal{M}(z) \mathcal{Y}_{n}(z) d z .
$$

Using the orthogonality relation, equation B 9, we obtain

$$
\widetilde{C}_{n}(s)=\frac{M_{n}+\widetilde{E}_{n}(s)}{s+\frac{E}{P r} \mu_{n}},
$$

and hence

$$
M=\sum_{n=0}^{\infty} C_{n}(\tau) \mathcal{Y}_{n}(z)
$$

where the functions $C_{n}(\tau)$ are obtained as the inverse Laplace transforms of the functions $\widetilde{C}_{n}(s)$, i.e.

$$
C_{n}(\tau)=\mathcal{L}^{-1} \widetilde{C}_{n}(s)
$$

We can write $\widetilde{E}_{n}(s)$ as

$$
\begin{gathered}
\widetilde{E}_{n}(s)=\frac{E \mu_{n}}{s} \frac{1}{y_{n}^{2}} \int_{-1 / 2}^{1 / 2}\left[\int_{-1 / 2}^{z} \frac{\nu}{\kappa} \frac{\partial K_{0}}{\partial z^{\prime}} d z^{\prime}\right] \mathcal{Y}_{n} d z \\
+\sum_{m=0}^{\infty} B_{m}\left[\frac{E \lambda_{m}\left(s+E \lambda_{m}\right)-1}{\left(s+E \lambda_{m}\right)^{2}+1}\right] \frac{1}{y_{n}^{2}} \int_{-1 / 2}^{1 / 2} \mathcal{Z}_{m} \mathcal{Y}_{n} d z
\end{gathered}
$$

where we have used integration by parts on the first term to write

$$
\int_{-1 / 2}^{1 / 2} \frac{\partial}{\partial z}\left(\nu \frac{\partial K_{0}}{\partial z}\right) \mathcal{Y}_{n} d z=-\mu_{n} \int_{-1 / 2}^{1 / 2}\left[\int_{-1 / 2}^{z} \frac{\nu}{\kappa} \frac{\partial K_{0}}{\partial z^{\prime}} d z^{\prime}\right] \mathcal{Y}_{n} d z
$$

Hence

$$
\begin{aligned}
\widetilde{C}_{n}(s) & =\frac{M_{n}}{s+\frac{E}{P r} \mu_{n}}+\frac{E \mu_{n}}{s\left(s+\frac{E}{P r} \mu_{n}\right)} \frac{1}{y_{n}^{2}} \int_{-1 / 2}^{1 / 2}\left[\int_{-1 / 2}^{z} \frac{\nu}{\kappa} \frac{\partial K_{0}}{\partial z^{\prime}} d z^{\prime}\right] \mathcal{Y}_{n} d z \\
& +\sum_{m=0}^{\infty} B_{m}\left[\frac{E \lambda_{m}\left(s+E \lambda_{m}\right)-1}{\left(s+\frac{E}{P r} \mu_{n}\right)\left(\left(s+E \lambda_{m}\right)^{2}+1\right)}\right] \frac{1}{y_{n}^{2}} \int_{-1 / 2}^{1 / 2} \mathcal{Z}_{m} \mathcal{Y}_{n} d z
\end{aligned}
$$

and so

$$
\begin{aligned}
C_{n}(\tau) & =M_{n} e^{-\frac{E}{P r} \mu_{n} \tau}+\operatorname{Pr}\left[1-e^{-\frac{E}{P r} \mu_{n} \tau}\right] \frac{1}{y_{n}^{2}} \int_{-1 / 2}^{1 / 2}\left[\int_{-1 / 2}^{z} \frac{\nu}{\kappa} \frac{\partial K_{0}}{\partial z^{\prime}} d z^{\prime}\right] \mathcal{Y}_{n} d z \\
+ & \sum_{m=0}^{\infty} B_{m}\left[\frac{1}{y_{n}^{2}} \int_{-1 / 2}^{1 / 2} \mathcal{Z}_{m} \mathcal{Y}_{n} d z\right]\left[\frac{E^{2} \lambda_{m}\left(\lambda_{m}-\frac{\mu_{n}}{P r}\right)-1}{E^{2}\left(\lambda_{m}-\frac{\mu_{n}}{P r}\right)^{2}+1} e^{-\frac{E}{P r} \mu_{n} \tau}\right. \\
+ & \left.\frac{\left(1-E^{2} \lambda_{m}\left(\lambda_{m}-\frac{\mu_{n}}{P r}\right)\right) \cos (\tau)+E\left(2 \lambda_{m}-\frac{\mu_{n}}{P r}\right) \sin (\tau)}{E^{2}\left(\lambda_{m}-\frac{\mu_{n}}{P r}\right)^{2}+1} e^{-E \lambda_{m} \tau}\right] .
\end{aligned}
$$


The general solution for $M$ can now be written as

$$
\begin{gathered}
M(z, \tau)=\sum_{n=0}^{\infty} M_{n} \mathcal{Y}_{n}(z) e^{-\frac{E}{P r} \mu_{n} \tau}+\operatorname{Pr} \int_{-1 / 2}^{z} \frac{\nu}{\kappa} \frac{\partial K_{0}}{\partial z^{\prime}} d z^{\prime} \\
-\operatorname{Pr} \sum_{n=0}^{\infty}\left[\frac{1}{y_{n}^{2}} \int_{-1 / 2}^{1 / 2}\left(\int_{-1 / 2}^{z} \frac{\nu}{\kappa} \frac{\partial K_{0}}{\partial z^{\prime}} d z^{\prime}\right) \mathcal{Y}_{n}(z) d z\right] \mathcal{Y}_{n}(z) e^{-\frac{E}{P r} \mu_{n} \tau} \\
+\sum_{n=0}^{\infty} \sum_{m=0}^{\infty} B_{m}\left[\frac{1}{y_{n}^{2}} \int_{-1 / 2}^{1 / 2} \mathcal{Z}_{m}(z) \mathcal{Y}_{n}(z) d z\right]\left[\frac{E^{2} \lambda_{m}\left(\lambda_{m}-\frac{\mu_{n}}{P r}\right)-1}{E^{2}\left(\lambda_{m}-\frac{\mu_{n}}{P r}\right)^{2}+1} e^{-\frac{E}{P r} \mu_{n} \tau}\right. \\
\left.+\frac{\left(1-E^{2} \lambda_{m}\left(\lambda_{m}-\frac{\mu_{n}}{P r}\right)\right) \cos (\tau)+E\left(2 \lambda_{m}-\frac{\mu_{n}}{P r}\right) \sin (\tau)}{E^{2}\left(\lambda_{m}-\frac{\mu_{n}}{P r}\right)^{2}+1} e^{-E \lambda_{m} \tau}\right] \mathcal{Y}_{n}(z) .
\end{gathered}
$$

The first term of equation B 20 corresponds to adjustment from the initial state, the second and third correspond to adjustment towards the final steady state solution and the last double sum term represents the effects of the velocity adjustment with the $\cos \tau$ and $\sin \tau$ terms describing the inertial waves generated during the adjustment phase.

When $\nu=\kappa$ equations 3.21 and B 8 have the same form and hence $\mathcal{Z}_{n}=\mathcal{Y}_{n}, \lambda_{n}=\mu_{n}$ and $z_{n}=y_{n}$. So we can write

$$
\begin{gathered}
C_{n}(\tau)=\frac{P r}{z_{n}^{2}}\left[1-e^{-\frac{E}{P r} \lambda_{n} \tau}\right]\left[\int_{-1 / 2}^{1 / 2} K_{0} \mathcal{Z}_{n} d z\right]+B_{n}\left[\frac{E^{2} \lambda_{n}^{2}\left(1-\frac{1}{P r}\right)-1}{E^{2} \lambda_{n}^{2}\left(1-\frac{1}{P r}\right)^{2}+1} e^{-\frac{E}{P r} \lambda_{n} \tau}\right. \\
\left.+\frac{\left(1-E^{2} \lambda_{n}^{2}\left(1-\frac{1}{P r}\right)\right) \cos \tau+E \lambda_{n}\left(2-\frac{1}{P r}\right) \sin \tau}{E^{2} \lambda_{n}^{2}\left(1-\frac{1}{P r}\right)^{2}+1} e^{-E \lambda_{n} \tau}\right]+M_{n} e^{-\frac{E}{P r} \lambda_{n} \tau}
\end{gathered}
$$

For $\operatorname{Pr}=1$ this becomes

$$
C_{n}(\tau)=\frac{1}{z_{n}^{2}}\left[1-e^{-E \lambda_{n} \tau}\right]\left[\int_{-1 / 2}^{1 / 2} K_{0} \mathcal{Z}_{n} d z\right]+\left[\left(B_{n} \cos \tau+E \lambda_{n} \sin \tau-1\right)+M_{n}\right] e^{-E \lambda_{n} \tau} \text {. }
$$

\section{Appendix C. Numerical Solutions for the Structure Functions}

For general $\nu$ and $\kappa$ we need to solve for the functions $K_{0}, \mathcal{Z}_{n}$ and $\mathcal{Y}_{n}$ and eigenvalues $\lambda_{n}$ and $\mu_{n}$ numerically. The equations and boundary conditions are

$$
\begin{cases}\left(\nu\left(\nu K_{0}^{\prime}\right)^{\prime \prime}\right)^{\prime}=-K_{0}-\zeta, & \zeta \in[-1 / \sqrt{4 E}, 1 / \sqrt{4 E}] \\ \left(\nu K_{0}^{\prime}\right)^{\prime \prime}=0, & \zeta= \pm 1 / \sqrt{4 E} \\ K_{0}^{\prime}=0, & \zeta= \pm 1 / \sqrt{4 E}\end{cases}
$$

for $K_{0}(\zeta)$ and

$$
\begin{cases}\left(\nu \mathcal{Z}_{n}^{\prime}\right)^{\prime}=-\lambda_{n} \mathcal{Z}_{n}, & z \in[-1 / 2,1 / 2], \\ \mathcal{Z}_{n}^{\prime}=0, & z= \pm 1 / 2, \\ \mathcal{Z}_{n}=1, & z=1 / 2\end{cases}
$$

and

$$
\begin{cases}\left(\kappa \mathcal{Y}_{n}^{\prime}\right)^{\prime}=-\mu_{n} \mathcal{Y}_{n}, & z \in[-1 / 2,1 / 2] \\ \mathcal{Y}_{n}^{\prime}=0, & z= \pm 1 / 2, \\ \mathcal{Y}_{n}=1, & z=1 / 2\end{cases}
$$


for $\mathcal{Z}_{n}(z)$ and $\mathcal{Y}_{n}(z)$. We choose the boundary conditions $\mathcal{Z}_{n}, \mathcal{Y}_{n}=1$ at $z=1 / 2$ to normalise our eigenfunctions. The constants $z_{n}$ and $y_{n}$ can be calculated by numerical integration using the solutions.

We now consider a function $\mathbf{g}(\boldsymbol{\sigma}) \in \mathbb{R}^{m}$ where $\boldsymbol{\sigma} \in \mathbb{R}^{m}$ for some positive integer $m$. We seek the roots of $\mathbf{g}$, i.e. the solutions $\boldsymbol{\sigma}=\boldsymbol{\sigma}^{*}$ satisfying $\mathbf{g}(\boldsymbol{\sigma})=\mathbf{0}$. We can solve for $\boldsymbol{\sigma}^{*}$ numerically using the Newton-Raphson iteration

$$
\boldsymbol{\sigma}_{n+1}=\boldsymbol{\sigma}_{n}-\left[\frac{\partial \mathbf{g}}{\partial \boldsymbol{\sigma}}\left(\boldsymbol{\sigma}_{n}\right)\right]^{-1} \cdot \mathbf{g}\left(\boldsymbol{\sigma}_{n}\right),
$$

for some suitable chosen initial guess $\boldsymbol{\sigma}_{0}$. The Jacobian matrix, given by

$$
\left[\frac{\partial \mathbf{g}}{\partial \boldsymbol{\sigma}}\right]_{i j}=\frac{\partial g_{i}}{\partial \sigma_{j}}
$$

can be approximated numerically to second order by

$$
\left[\frac{\partial \mathbf{g}}{\partial \boldsymbol{\sigma}}\right]_{i j} \approx \frac{1}{2 \delta}\left[g_{i}\left(\boldsymbol{\sigma}+\delta \mathbf{e}_{j}\right)-g_{i}\left(\boldsymbol{\sigma}-\delta \mathbf{e}_{j}\right)\right],
$$

for some small $\delta$ requiring $2 m$ evaluations of $\mathbf{g}$. We now iterate equation $\mathrm{C} 4$ until a suitable convergence condition is met, $\boldsymbol{\sigma}_{0}$ is chosen near the root such that the method converges. We say that the method has converged once

$$
\left|\mathbf{g}\left(\boldsymbol{\sigma}_{n}\right)\right|<\epsilon^{*}
$$

for small parameter $\epsilon^{*}$.

We now write equations C 1 - C 3 as first order systems of the form

$$
\mathbf{y}^{\prime}=\mathbf{h}(\mathbf{y}, z)
$$

for solution vector $\mathbf{y}$, function $\mathbf{h}$ and parameter $z$. We write equation $\mathrm{C} 1$ as

$$
\left(\begin{array}{c}
K_{0} \\
\nu K_{0}^{\prime} \\
\left(\nu K_{0}^{\prime}\right)^{\prime} \\
\nu\left(\nu K_{0}^{\prime}\right)^{\prime \prime}
\end{array}\right)^{\prime}=\left(\begin{array}{c}
{\left[\nu K_{0}^{\prime}\right] / \nu} \\
\left(\nu K_{0}^{\prime}\right)^{\prime} \\
{\left[\nu\left(\nu K_{0}^{\prime}\right)^{\prime \prime}\right] / \nu} \\
-K_{0}-\zeta
\end{array}\right)
$$

with initial condition

$$
\left(\begin{array}{c}
K_{0} \\
\nu K_{0}^{\prime} \\
\left(\nu K_{0}^{\prime}\right)^{\prime} \\
\nu\left(\nu K_{0}^{\prime}\right)^{\prime \prime}
\end{array}\right)=\left(\begin{array}{c}
\sigma_{1} \\
0 \\
\sigma_{2} \\
0
\end{array}\right)
$$

at $\zeta=-1 / \sqrt{4 E}$. We now integrate from $\zeta=-1 / \sqrt{4 E}$ to $\zeta=1 / \sqrt{4 E}$ using a RungeKutta method and define

$$
\mathbf{g}\left(\sigma_{1}, \sigma_{2}\right)=\left.\left(\begin{array}{c}
\nu K_{0}^{\prime} \\
\nu\left(\nu K_{0}^{\prime}\right)^{\prime \prime}
\end{array}\right)\right|_{\zeta=1 / \sqrt{4 E}}
$$

We can see that finding a numerical solution for $K_{0}$ requires us to calculate the correct initial conditions $\left(\sigma_{1}, \sigma_{2}\right)$ such that the boundary conditions at $\zeta=1 / \sqrt{4 E}$ are satisfied, these conditions correspond to $\mathbf{g}=\mathbf{0}$ so we can solve for $\left(\sigma_{1}, \sigma_{2}\right)$ iteratively using equation $\mathrm{C} 4$. The solution for $K_{0}$ is given by the solution using the value of $\boldsymbol{\sigma}_{n}$ once the convergence condition has been met. 


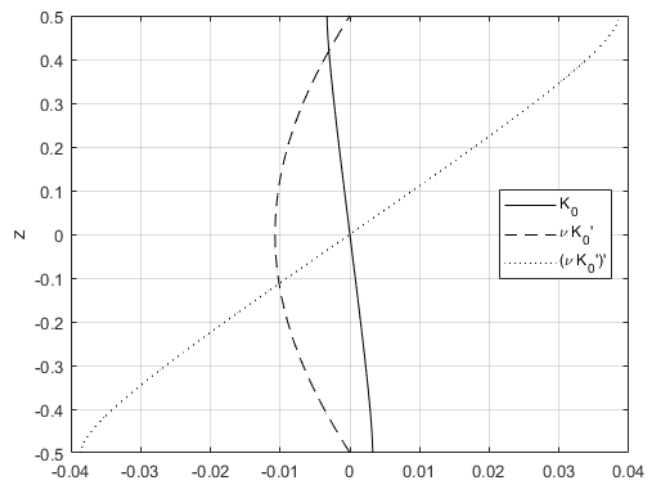

(a)

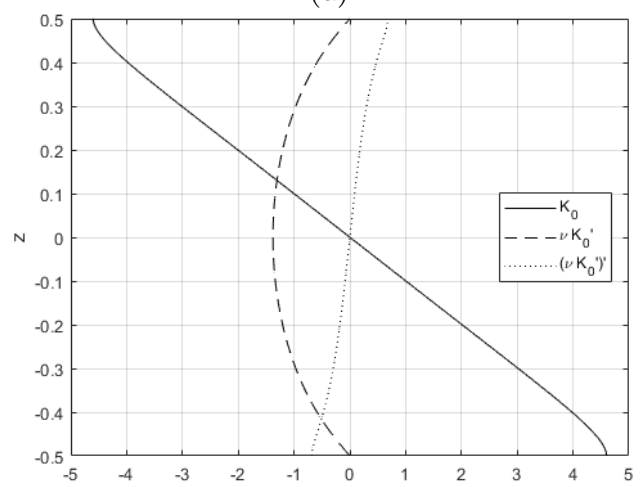

(c)



(b)

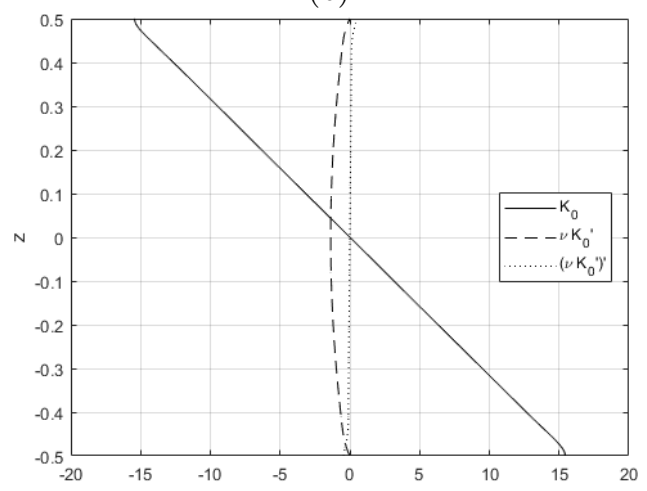

(d)

Figure 10: Vertical structure function, $K_{0}$ as a function of $z$ for $E=1$ (a), 0.1 (b), 0.01 (c) and $0.001(\mathrm{~d})$ and $\nu=11 / 8-9 / 2 z^{2}$.

We now write equations $\mathrm{C} 2$ and $\mathrm{C} 3$ as

$$
\left(\begin{array}{c}
\mathcal{Z}_{n} \\
\nu \mathcal{Z}_{n}^{\prime} \\
\mathcal{Y}_{n} \\
\kappa \mathcal{Y}_{n}^{\prime}
\end{array}\right)^{\prime}=\left(\begin{array}{c}
{\left[\nu \mathcal{Z}_{n}^{\prime}\right] / \nu} \\
-\lambda_{n} \mathcal{Z}_{n} \\
{\left[\kappa \mathcal{Y}_{n}^{\prime}\right] / \kappa} \\
-\mu_{n} \mathcal{Y}_{n}
\end{array}\right)
$$

with initial condition

$$
\left(\begin{array}{c}
\mathcal{Z}_{n} \\
\nu \mathcal{Z}_{n}^{\prime} \\
\mathcal{Y}_{n} \\
\kappa \mathcal{Y}_{n}^{\prime}
\end{array}\right)=\left(\begin{array}{l}
1 \\
0 \\
1 \\
0
\end{array}\right)
$$

at $z=1 / 2$ and integrate backwards in $z$. In order to satisfy the boudary conditions at $z=-1 / 2$ we define

$$
\mathbf{g}\left(\sigma_{1}, \sigma_{2}\right)=\left.\left(\begin{array}{c}
\nu \mathcal{Z}_{n}^{\prime} \\
\kappa \mathcal{Y}_{n}^{\prime}
\end{array}\right)\right|_{z=-1 / 2},
$$

where $\left(\sigma_{1}, \sigma_{2}\right)=\left(\lambda_{n}, \mu_{n}\right)$ and take $\boldsymbol{\sigma}$ such that $\mathbf{g}=\mathbf{0}$. Therefore the solution $\boldsymbol{\sigma}=\boldsymbol{\sigma}^{*}$ determines the eigenvalues so we expect the components of $\mathbf{g}$ to be oscillatory with infinitely many positive roots. We note that the first component of $\mathbf{g}$ depends only on $\lambda_{n}$ while the second depends only on $\mu_{n}$. We can plot $g_{1}\left(\lambda_{n}\right)$ and $g_{2}\left(\mu_{n}\right)$ and use the plots to 


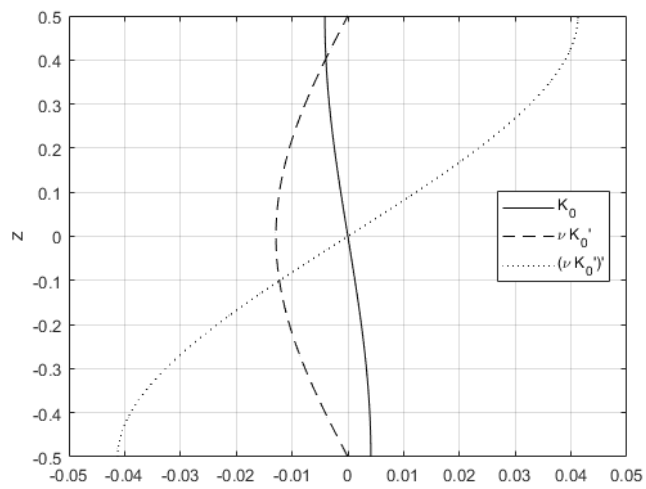

(a)

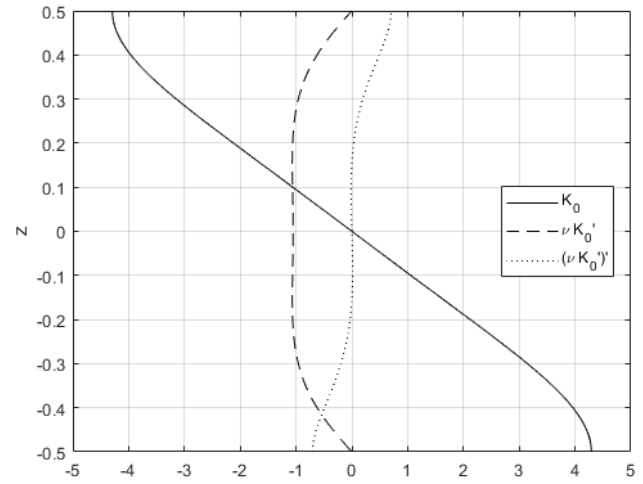

(c)

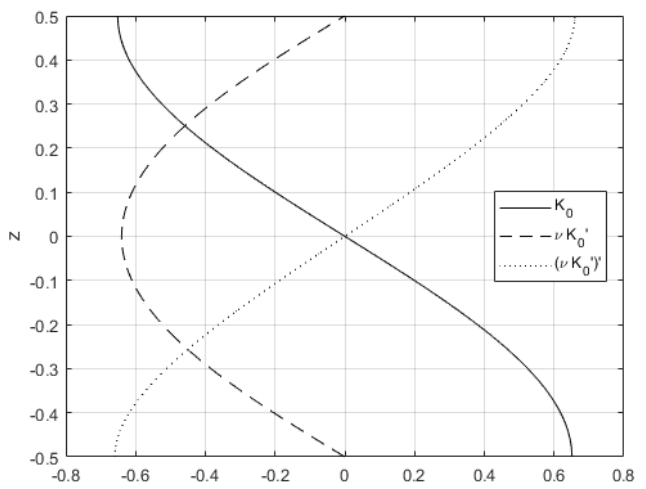

(b)

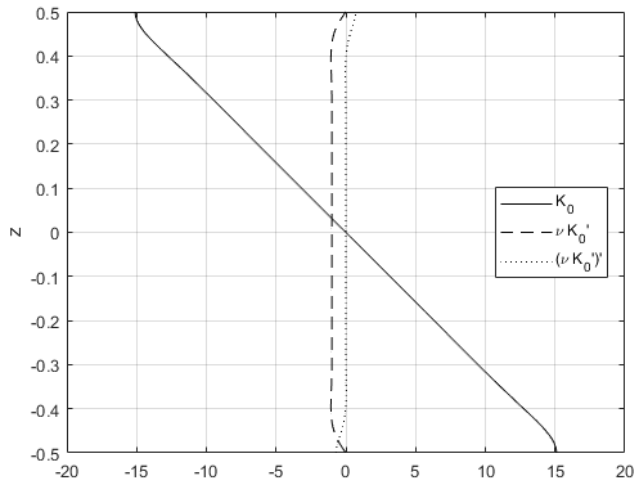

(d)

Figure 11: Vertical structure function, $K_{0}$ as a function of $z$ for for $E=1$ (a), 0.1 (b), 0.01 (c) and $0.001(\mathrm{~d})$ and $\nu=1$.

determine approximate values for the eigenvalues, these can be taken as starting values, $\boldsymbol{\sigma}_{0}$, in a Newton-Raphson iteration using equation C 4 . The solutions and eigenvalues are given once the method has converged.

We now consider a KPP-like profile for $\kappa=\nu$ where $\nu$ is given by

$$
\nu=\frac{11}{8}-\frac{9}{2} z^{2}
$$

Numerical solutions for $K_{0}$ with $E=1,0.1,0.01$ and 0.001 are shown in Figure 10. We can see that the upwelling, which is proportional to $\nu K_{0}^{\prime}$, is stronger for smaller $E$ and in the small $E$ limit the along front velocity, which is proportional to $K_{0}^{\prime}$, approaches the linear Thermal Wind profile. The cross front velocity, proportional to $\left(\nu K_{0}^{\prime}\right)^{\prime}$, is greatest at the surface and for small $E$ is confined to thin boundary layers near the surfaces.

Figure 11 shows the solutions for $K_{0}$ with $E=1,0.1,0.01$ and 0.001 in the case where $\nu=1$, we can see that the form of the solutions is similar to the KPP case with a high horizontal surface velocity and strong upwelling in the centre of the vertical domain. For small $E$, the boundary layers are thicker for the constant case as the viscosity in this region is higher and the upwelling is approximately constant throughout the vertical domain. In both cases the along front velocity approaches the linear Thermal Wind profile for small $E$.

Figure 12 shows a comparison between the first eight vertical harmonics, $\mathcal{Z}_{n}$, for the 


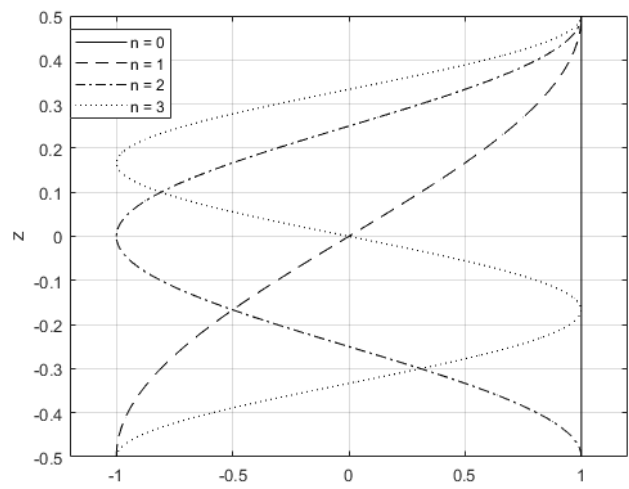

(a)

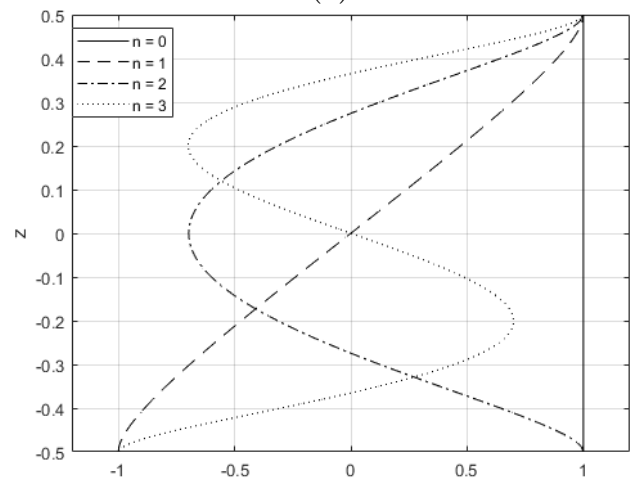

(c)

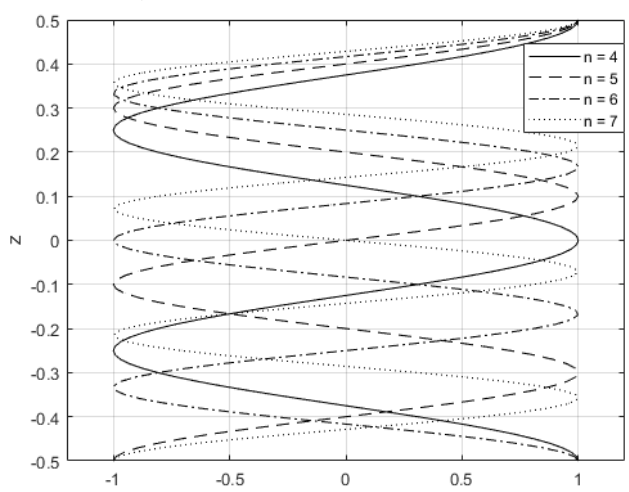

(b)

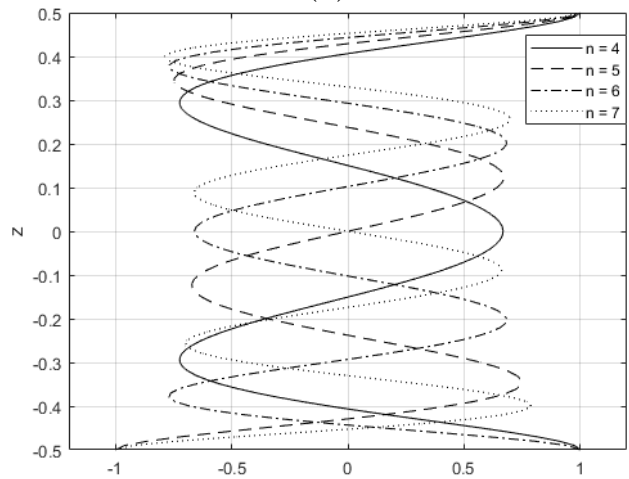

(d)

Figure 12: (a) $\mathcal{Z}_{n}$ for $\nu=1$ and $n \in\{0,1,2,3\}$. (b) $\mathcal{Z}_{n}$ for $\nu=1$ and $n \in\{4,5,6,7\}$. (c) $\mathcal{Z}_{n}$ for $\nu=11 / 8-9 / 2 z^{2}$ and $n \in\{0,1,2,3\}$. (d) $\mathcal{Z}_{n}$ for $\nu=11 / 8-9 / 2 z^{2}$ and $n \in\{4,5,6,7\}$. We note that if $\kappa=\nu$ then $\mathcal{Y}_{n}=\mathcal{Z}_{n}$.

constant and KPP viscosity profiles. We note that $\mathcal{Y}_{n}=\mathcal{Z}_{n}$ when $\kappa=\nu$. We can see that the magnitude of the osillations in the interior is smaller for the KPP case as the viscosity is higher here compared with the region near the boundary, we can think of this as stronger damping.

\section{REFERENCES}

Blumen, W. 2000 Inertial oscillations and frontogenesis in a zero potential vorticity model. $J$. Phys. Oceanogr. 30, 31-39.

Charney, J. G. 1973 Planetary Fluid Dynamics, chap. Symmetric Circulations in Idealized Models, pp. 128-141. D. Reidel Publishing Company.

Cronin, Meghan F \& Kessler, William S 2009 Near-surface shear flow in the tropical pacific cold tongue front. Journal of Physical Oceanography 39 (5), 1200-1215.

D'Asaro, Eric, Lee, Craig, Rainville, Luc, Harcourt, Ramsey \& Thomas, Leif 2011 Enhanced turbulence and energy dissipation at ocean fronts. science 332 (6027), 318-322.

Eliassen, A. 1962 On the vertical circulation in frontal zones. Geofys. Publ. 24 (4), 147-160.

ENRIQUEZ, R. M. \& TAYLOR, J. R. 2015 Shutdown of turbulent convection as a new criterion for the onset of spring phytoplankton blooms. ICES J. Mar. Sci. Fsv071.

Erdogan, M. E. \& Chatwin, P. C. 1967 The effects of curvature and buoyancy on the laminar dispersion of solute in a horizontal tube. J. Fluid Mech. 29, 465-484.

FERrari, R. 2011 A frontal challenge for climate models. Science 332 (6027), 316-317. 
FERrari, R. \& Young, W. R. 1997 On the development of thermohaline correlations as a result of nonlinear diffusive parameterizations. J. Mar. Res. 55, 1069-1101.

Garrett, C. J. R. \& Loder, J. W. 1981 Dynamical aspects of shallow sea fronts. Phil. Trans. R. Soc. Lond. A 302, 563-581.

Gula, J., Molemaker, M. J. \& McWilliams, J. C. 2014 Submesoscale cold filaments in the gulf stream. J. Phys. Oceanogr. 44, 2617-2643.

Holton, James R \& Hakim, Gregory J 2012 An introduction to dynamic meteorology, , vol. 88. Academic press.

Hoskins, B. J. 1982 The mathematical theory of frontogenesis. Annu. Rev. Fluid Mech. 14, $131-151$.

Hoskins, B. J. \& Bretherton, F. P. 1972 Atmospheric frontogenesis models: Mathematical formulation and solution. J. Atmos. Sci. 29, 11-37.

Johnston, TM, Rudnick, Daniel L \& Pallàs-Sanz, E 2011 Elevated mixing at a front. Journal of Geophysical Research: Oceans 116 (C11).

McWilliams, J. C. 2016 Submesoscale currents in the ocean. Proc. R. Soc. A 472.

McWilliams, J. C. 2017 Submesoscale surface fronts and filaments: secondary circulation, buoyancy flux, and frontogenesis. J. Fluid Mech. 823, 391-432.

McWilliams, J. C., Gula, J., Molemaker, M. J., Renault, L. \& Shchepetkin, A. F. 2015 Filament frontogenesis by boundary layer turbulence. J. Phys. Oceanogr. 45, 1988-2005.

Orlanski, I. \& Ross, B. B. 1977 The circulation associated with a cold front: Part i: Dry case. J. Atmos. Sci. 34, 1619-1633.

Ostdiek, V. \& Blumen, W. 1997 A dynamic trio: Inertial oscillation, deformation frontogenesis, and the ekman-taylor boundary layer. J. Atmos. Sci. 54, 1490-1502.

Rudnick, D. L. \& Luyten, J. R. 1996 Intensive surveys of the azores front: 1. tracers and dynamics. J. Geophys. Res. 101, 923-939.

SALMON, Rick 1998 Lectures on geophysical fluid dynamics. Oxford University Press.

Shakespeare, C. J. \& TAYlor, J.R. 2013 A generalized mathematical model of geostrophic adjustment and frontogenesis: uniform potential vorticity. J. Fluid Mech. 736, 366-413.

Sмiтh, R. 1982 Similarity solutions of a non-linear diffusion equation. IMA J. App. Math. 28 (2), 149-160.

Sullivan, P. P. \& McWilliams, J. C. 2018 Frontogenesis and frontal arrest of a dense filament in the oceanic surface boundary layer. J. Fluid Mech. 837, 1341-380.

TAYLOR, J. R. 2016 The influence of submesoscale restratification and convection on lightlimited phytoplankton growth. Geophys. Res. Lett. 2016GL069106.

TAYlor, J. R. \& Ferrari, R. 2010 Buoyancy and wind-driven convection at mixed layer density fronts. J. Phys. Oceanogr. 40, 1222-1242.

TAYlor, J. R. \& FERRARI, R. 2011 Numerical simulations of the competition between winddriven mixing and surface heating in triggering spring phytoplankton blooms. Limnol. Oceanogr. 56 (6), 2293-2307.

Thomas, Leif N, Taylor, John R, Ferrari, Raffaele \& Joyce, Terrence M 2013 Symmetric instability in the gulf stream. Deep Sea Research Part II: Topical Studies in Oceanography 91, 96-110.

Thompson, LuAnne 2000 Ekman layers and two-dimensional frontogenesis in the upper ocean. J. Geophys. Res.: Oceans 105 (C3), 6437-6451.

Wenegrat, Jacob O \& McPhaden, Michael J 2016 Wind, waves, and fronts: Frictional effects in a generalized ekman model. Journal of Physical Oceanography 46 (2), 371-394.

Young, W. R. 1994 The subinertial mixed layer approximation. J. Phys. Oceanogr. 24, 18121826. 